\title{
Adaptive Asymptotic Tracking Control for a Class of Uncertain Input-Delayed Systems with Periodic Time-Varying Disturbances
}

\author{
Xiaoman Yan, Chunsheng Zhang, Dewen Cao, and Jian Wu \\ University Key Laboratory of Intelligent Perception and Computing of Anhui Province, Anqing Normal University, \\ Anqing 246133, China \\ Correspondence should be addressed to Jian Wu; jwu2011@126.com
}

Received 14 December 2020; Revised 5 March 2021; Accepted 25 March 2021; Published 7 April 2021

Academic Editor: Defeng He

Copyright ( 2021 Xiaoman Yan et al. This is an open access article distributed under the Creative Commons Attribution License, which permits unrestricted use, distribution, and reproduction in any medium, provided the original work is properly cited.

In this paper, the problem of adaptive asymptotic tracking control for a class of uncertain systems with periodic time-varying disturbances and input delay is studied. By combining Fourier series expansion (FSE) with radial basis function neural network (RBFNN), a hybrid function approximator is used to learn the functions with periodic time-varying disturbances. At the same time, the dynamic surface control technique with a nonlinear filter is used to avoid the "complexity explosion" problem in the process of traditional backstepping technology. Ultimately, all closed-loop signals are guaranteed to be semiglobally uniformly bounded, and the given reference signal can be asymptotically tracked by the output signals of system. A simulation example is given to verify the effectiveness of the proposed control scheme.

\section{Introduction}

In recent years, with the deepening of the research on uncertain nonlinear control systems, more and more scholars have carried out indepth research on this problem and achieved remarkable results. The uncertainties appear in nonlinear systems in strict-feedback form [1-6], purefeedback form [7, 8], multiple-input-multiple-output (MIMO) form [9-11], time-delay form [12, 13], switched form [14-19], and discrete-time form [20, 21]. Aiming at different forms of system, different methods are proposed to solve the problem of system control. For example, the adaptive gain scheduling backstepping sliding mode control method [1] is proposed for a class of strict-feedback systems. The problem of predetermined performance control for a class of pure-feedback uncertain nonlinear systems with input saturation [7] is studied. For a class of uncertain MIMO nonlinear systems with given tracking performance, the error-driven nonlinear feedback design method [10] is proposed to improve the dynamic performance of fuzzy adaptive moving surface control. The dynamic output feedback fault-tolerant controller [12] is designed to solve the problems of fault estimation and fault-tolerant controller design for a class of discrete-time fuzzy systems. The adaptive fuzzy tracking control for a class of switched uncertain nonlinear systems [14] is studied. For a class of uncertain strict-feedback nonlinear systems, the output feedback adaptive neural tracking control [15] is studied.

In particular, the study of uncertain nonlinear systems with periodic perturbations is also a challenging problem [22-26]. Since RBFNN does not use periodic perturbation functions as inputs, it is difficult to deal with such systems in traditional ways. Therefore, some new methods are proposed to solve this problem. In particular, the study of uncertain nonlinear systems with periodic perturbations is also a challenging problem [27] is studied. This article [28] investigates the sampled data stabilization problem of a class of switched nonlinear systems. To guarantee that all states of the closed-loop system (CLS) are bounded, a new allowable sampling period is deduced.

It is well known that the traditional backstepping technology has the problem of "complexity explosion." To avoid the problem, many scholars have introduced dynamic surface control technology and achieved some results [29-34]. For a class of fractional-order nonlinear systems with uncertain parameters, the design of adaptive dynamic 
surface control [30] is studied. For a class of single input single output strict-feedback fractional-order uncertain nonlinear systems, the dynamic surface control method based on fractional-order filter [32] is proposed to avoid the inherent "complexity explosion" problem in the backstepping design process. The problem of adaptive fuzzy dynamic surface control [33] is studied for a class of nonstrict feedback nonlinear systems with unknown virtual control coefficients and full state constraints. The dynamic surface control technology [34] is used to solve the "complexity explosion" problem and combines the Nussbaum function to solve the problem of unknown control direction.

It is worth noting that, for uncertain systems with periodic perturbations, the asymptotic tracking control problem is not well solved. It is because the asymptotic tracking control problem of uncertain systems with periodic perturbations has not been well solved. Aiming at this difficulty, the control method proposed in this paper solves the asymptotic tracking control problem of this kind of system well. The main work is as follows: (1) in this paper, the problem of adaptive asymptotic tracking control for a class of nonlinear uncertain systems with periodic timevarying disturbances and input delay is studied. To solve this problem, we propose a new adaptive control scheme. (2) To ensure the feasibility of the control scheme, the FSE-RBFNN approximator is used to approximate the unknown disturbance function. (3) An adaptive dynamic surface control technique with a nonlinear filter is designed to avoid the "complexity explosion" problem in the traditional backstepping design process. (4) Based on the designed adaptive controller, all closed-loop signals are ultimately guaranteed to be semiglobally uniformly bounded. It is proved that the output signal can asymptotically track the given reference signal. Finally, a simulation example is given to verify the effectiveness of the proposed control scheme.

\section{Problem Description and Preliminaries}

A class of periodic perturbed nonlinear systems with strictfeedback is considered:

$$
\left\{\begin{array}{l}
\dot{x}_{i}=x_{i+1}+f_{i}\left(\bar{x}_{i}, \omega_{i}(t)\right)+d_{i}(t), \quad i=1, \ldots, n-1, \\
\dot{x}_{n}=u(t-\tau(t))+f_{n}\left(\bar{x}_{n}, \omega_{n}(t)\right)+d_{n}(t), \\
y=x_{1}
\end{array}\right.
$$

for $i=1, \ldots, n, \bar{x}_{i}=\left[x_{1}, \ldots, x_{i}\right]^{T}$ is the system state variable, $y \in R$ is the output of the system, $u$ is the control input of the system, $\tau(t)$ represents the time-varying delay, $f_{i}(\cdot): \mathbb{R}^{i+q_{i}} \longrightarrow \mathbb{R}$ is the unknown continuous function, $\omega_{i}(t)$ is the unknown continuous time-varying disturbance with known period $T_{i}$, i.e., $\omega_{i}\left(t+T_{i}\right)=\omega_{i}(t),[0,+\infty)$ $\longrightarrow \mathbb{R}^{q_{i}}$, and $d_{i}(t)$ is the disturbance function.

Remark 1. For the actual control systems, such as industrial control systems, communication systems, and aerospace control systems, the disturbance and time-delay are common problems. These problems are well solved, and the performance of the controlled systems will have a great improvement.

Control objective: an adaptive dynamic surface control algorithm based on a neural network is designed to ensure that the output signal of system (1) can track the reference signal $y_{d}(t)$ asymptotically, and all the closed-loop signals are semiglobally uniformly bounded.

To achieve the above control objectives, we make the following assumptions on system (1):

Assumption 1 (see [32]): reference trajectory $y_{d}(t)$ is bounded, and its derivatives $\dot{y}_{d}(t)$ and $\ddot{y}_{d}(t)$ are continuous and bounded

Assumption 2 (see [35]): for $i=1,2, \ldots, n$, there exists an unknown constant $N_{i}$, which makes the following inequality holds $\left|d_{i}(t)\right| \leq N_{i}$

In this paper, the mixed function approximator based on FSE-RBFNN in reference [28] is used to model $g_{i}\left(E_{i}, \omega_{i}(t)\right)$ as follows:

$$
g_{i}\left(E_{i}, \omega_{i}(t)\right)=W^{T} S\left(E_{i}, \gamma^{T} \varphi(t)\right)+\delta\left(E_{i}, t\right) .
$$

A hybrid neural network approximator is designed

$$
\begin{aligned}
H\left(E_{i}, t\right) & =W^{T} S\left(E_{i}, \gamma^{T} \varphi(t)\right), \\
\delta\left(E_{i}, t\right)= & W^{T} S\left(E_{i}, \gamma^{T} \varphi(t)+\delta_{\omega}(t)\right)-W^{T} S\left(E_{i}, \gamma^{T} \varphi(t)\right) \\
& +\delta_{b}\left(E_{i}, \omega(t)\right), \\
\left|\delta_{\omega}(t)\right| \leq & \varepsilon_{\omega}, \\
\left|\delta_{b}\left(E_{i}, \omega(t)\right)\right| \leq & \varepsilon_{b},
\end{aligned}
$$

where $E_{i} \subset \Omega_{E} \subset \mathbb{R}^{l}$ is a measured signal, $\omega_{i}(t)$ is an unknown continuous disturbance vector with known period $T$, $\omega_{i}(t)=\left[v_{1}(t), \ldots, v_{q}(t)\right] \in \Omega_{\omega} \subset \mathbb{R}^{q}, \Omega_{E}$ and $\Omega_{\omega}$ are compact sets, optimal weight vector $W=\operatorname{argmin}_{\widehat{w} \in \mathbb{R}^{h}}\left\{\sup _{\left(E_{i}, \omega(t)\right)}\right.$ $\left.\in \Omega\left|g\left(E_{i}, \omega(t)\right)-\widehat{W}^{T} S\left(E_{i}, \omega(t)\right)\right|\right\}, \quad W=\left[\omega_{1}, \ldots, \omega_{h}\right]^{T}, \quad \delta_{b}$ $\left(E_{i}, \omega(t)\right)$ is the inherent NN approximation error with the minimum upper bound $\varepsilon_{b}>0$, which can be decreased by increasing the $\mathrm{NN}$ node number $p, \quad S\left(E_{i}, \omega(t)\right)$ $=\left[s_{1}\left(E_{i}, \omega(t)\right), \ldots, s_{h}\left(E_{i}, \omega(t)\right)\right]$ is a known smooth vector value function, its component $s_{i}\left(E_{i}, \omega(t)\right)=\exp$ $\left[-\left\|\zeta-v_{i}\right\|^{2} / \zeta^{2}\right](i=1, \ldots, h), \zeta=\left[E_{i}^{T}, \omega^{T}(t)\right]^{T}, v_{i} \in \Omega$ is the center of $S\left(E_{i}, \omega(t)\right), \zeta>0$ is the width of $S\left(E_{i}, \omega(t)\right), \gamma=$ $\left[\gamma_{1}, \ldots, \gamma_{q}\right] \in \mathbb{R}^{s \times q}$ is a constant matrix, $\gamma_{i}$ is a vector composed of $s$ coefficients expanded by fourier series ( $s$ is an odd number), $\varphi(t)=\left[\varphi_{1}(t), \ldots, \varphi_{s}(t)\right]^{T}, \quad \varphi_{1}(t)=1$, $\varphi_{2 j}(t)=\sqrt{2} \sin (2 \pi j t / T)$, and $\varphi_{2 j+1}(t)=\sqrt{2} \cos (2 \pi j t / T)$, $j=1, \ldots,(s-1) / 2$, its $n$-th derivative is smooth and bounded, the upper bound $\varepsilon_{\omega}$ is the minimum truncation error of $\delta_{\omega}(t)$.

Lemma 1 (see [36]). For $\left(E_{i}, \omega(t)\right) \in \Omega$, the approximation error $\delta_{b}\left(E_{i}, t\right)$ in (2) satisfies the following condition:

$$
\left|\delta\left(E_{i}, t\right)\right| \leq \varepsilon
$$


where $\varepsilon$ is the minimum upper bound of $\delta\left(E_{i}, t\right)$ and it can be reduced arbitrarily by increasing the values of $s$ and $h$.

Generally speaking, the parameters $W$ and $\gamma$ are unknown. It needs to be estimated in the controller design. Suppose $\widehat{W}$ and $\widehat{\gamma}$ are, respectively, the estimates of $W$ and $\gamma$, and the estimated error is $\widetilde{W}=W-\widehat{W}, \widetilde{\gamma}=\gamma-\widehat{\gamma}$.

Lemma 2 (see [36]). For approximator (2), the estimated error can be expressed as follows:

$$
\begin{aligned}
W^{T} S\left(E_{i}, \gamma^{T} \varphi(t)\right)-\widehat{W}^{T} S\left(E_{i}, \widehat{\gamma}^{T} \varphi(t)\right)= & \widetilde{W}^{T}\left(\widehat{S}-\widehat{S}^{\prime} \widehat{\gamma}^{T} \varphi(t)\right) \\
& +\widehat{W}^{T} \widehat{S}^{\prime} \widetilde{\gamma}^{T} \varphi(t)+b,
\end{aligned}
$$

where $\widehat{S}=S\left(E_{i}, \widehat{\gamma}^{T} \varphi(t)\right), \widehat{S}^{\prime}=\left[\widehat{s}_{1}^{\prime}, \ldots, \widehat{s}_{h}^{\prime}\right] \in \mathbb{R}^{q \times h}$, and $\widehat{s}_{i}^{\prime}=$ $\left(\partial s_{i}\left(E_{i}, \omega\right)\right) /\left.\partial \omega\right|_{\omega=\hat{\gamma}^{T} \varphi(t)}, i=1, \ldots, h$. The remainder $b$ is bounded

$$
b \leq\|\gamma\|_{F}\left\|\varphi(t) \widehat{W}^{T} \widehat{S}^{\prime}\right\|_{F}+\|W\|\left\|\varphi(t) \widehat{\gamma}^{T} \widehat{S}^{\prime}\right\|_{F}+|W|_{1} .
$$

Lemma 3 (see [37]). For $\varsigma>0$ and $\lambda \in \mathbb{R}$, the following inequality holds:

$$
0 \leq|\lambda|-\frac{\lambda^{2}}{\sqrt{\lambda^{2}+\varsigma^{2}}} \leq \varsigma
$$

Lemma 4 (see [38]). Assume that $a$ is an unknown nonzero variable. $V\left(t_{0}, t\right)$ and $R(t)$ are smooth functions defined in the time interval $\left[t_{0}, t\right)$, and $V\left(t_{0}, t_{f}\right)$ is nonnegative, $R\left(t_{0}\right)$ is bounded. In addition, $N(R(t))=e^{R^{2}(t)} R(t)$ is a dynamic gain function, $\xi$ is a normal number, and $\bar{\xi}$ is a bounded variable. If the following inequality holds

$$
V\left(t_{0}, t\right) \leq e^{-\xi t} \int_{t_{0}}^{t} a N(R(c)) \dot{R}(c) e^{\xi c} \mathrm{~d} c+e^{-\xi t} \int_{t_{0}}^{t} \dot{R}(c) e^{\xi c} \mathrm{~d} c+\bar{\xi},
$$

it can be concluded that $R(t), e^{-\xi t} \int_{t_{0}}^{t} \dot{R}(c) e^{\xi c} d c, V\left(t_{0}, t\right)$ and $e^{-\xi t} \int_{t}^{t} a N(R(c)) \dot{R}(c) e^{\xi c} d c$ must be bounded on the interval $\left[t_{0}, t_{f}\right)$. Furthermore, when $t_{f}=\infty$, the obtained closed-loop system is also bounded.

Lemma 5 (see [38]). For the following equation

$$
\dot{\hat{m}}(t)=-a \widehat{m}(t)+d \bar{k}(t)
$$

where $a>0$ and $d>0$ are the design constants, $\bar{k}(t)$ is a positive function. If the initial value $\widehat{m}(0)$ is nonnegative, then for any $t>0, \widehat{m}(t) \geq 0$ must be established.

\section{Control Design and Stability Analysis}

The main conclusions of this paper can be summarized as the following theorem.

Theorem 1. Consider a closed-loop system (1), including the nonlinear filter (10), the actual controller (20), and the adaptive law (29). Based on Assumption 1 and Assumption 2, for any initial condition satisfying $V(0) \leq c$, where $c$ is a positive design parameter, there exists design parameters $k_{i}, \eta_{i}, \beta_{i}, \xi_{j}(i=1, \ldots, n, j=1, \ldots, n-1)$ and invertible $m a-$ trices $\Gamma_{W_{i}}$ and $\Gamma_{\gamma_{i}}$, such that

(i) All the closed-loop signals remain semiglobally uniformly ultimate bounded (SGUUB)

(ii) The given reference signal can be asymptotically tracked by the output signal of the system

The proof of Theorem 1 is divided into two parts: adaptive controller design and stability analysis of the closed-loop system.

In the following, we propose an adaptive NN dynamic surface control scheme based on the backstepping design process. To order to avoid the "complexity explosion" problem, the following nonlinear filters are constructed:

$$
\begin{gathered}
\iota_{i} \dot{s}_{i}=-\iota_{i} e_{i}-\frac{\iota_{i} \hat{M}_{i}^{2} e_{i}}{\sqrt{\widehat{M}_{i}^{2} e_{i}^{2}+\mu^{2}}}, \\
s_{i}(0)=\alpha_{i}(0), \quad i=1, \ldots, n-1,
\end{gathered}
$$

where $e_{i}=s_{i}-\alpha_{i}$ is the $i$-th boundary layer error and $\iota_{i}$ and $\mu$ are the positive constants. To compensate for the influence of input delay, the following system [35] is introduced:

$$
\begin{aligned}
& \dot{\lambda}_{i}=\lambda_{i+1}-p_{i} \lambda_{i}, \quad i=2, \ldots, n-1, \\
& \dot{\lambda}_{n}=-p_{n} \lambda_{n}+u(t-\tau(t))-u(t),
\end{aligned}
$$

where $p_{i}>1(i=2, \ldots, n)$ is optional known parameter, and the initial condition of the system is $\lambda(0)=0$.

In the next part, an adaptive $\mathrm{NN}$ tracking controller is designed based on the backstepping method. Firstly, the following coordinate transformations are introduced:

$$
\begin{aligned}
& z_{1}=x_{1}-y_{d} \\
& z_{i}=x_{i}-s_{i-1}-\lambda_{i}, \quad i=2, \ldots, n-1 .
\end{aligned}
$$

Step 1: according to (12), we can get the following result:

$$
\begin{aligned}
\dot{z}_{1} & =\dot{x}_{1}-\dot{y}_{d} \\
& =x_{2}+f_{1}\left(\bar{x}_{1}, \omega_{1}(t)\right)+d_{1}(t)-\dot{y}_{d} \\
& =z_{2}+s_{1}+\lambda_{2}+f_{1}\left(\bar{x}_{1}, \omega_{1}(t)\right)+d_{1}(t)-\dot{y}_{d} .
\end{aligned}
$$

The Lyapunov function $V_{1}$ is constructed as follows:

$$
\begin{aligned}
V_{1}= & \frac{1}{2} z_{1}^{2}+\frac{1}{2 \eta_{1}} \tilde{\theta}_{1}^{2}+\frac{1}{2} \tilde{W}_{1}^{T} \Gamma_{w_{1}}^{-1} \tilde{W}_{1}+\frac{1}{2} \operatorname{tr}\left\{\tilde{\gamma}_{1}^{T} \Gamma_{\gamma_{1}}^{-1} \tilde{\gamma}_{1}\right\} \\
& +\frac{1}{2 \beta_{1}} \tilde{N}_{1}^{2},
\end{aligned}
$$

where $\eta_{1}>0$ and $\beta_{1}>0$ are the design parameters, the estimation errors $\widetilde{\theta}_{1}=\theta_{1}-\widehat{\theta}_{1}, \widetilde{N}_{1}=N_{1}-\widehat{N}_{1}, \widehat{\theta}_{1}$ and $\widehat{N}_{1}$ represent the estimation of $\theta_{1}$ and $N_{1}$, respectively. $\Gamma_{w_{1}}>0$ and $\Gamma_{\gamma_{1}}>0$ are the invertible matrices of the design. Then, we can get 


$$
\begin{gathered}
\dot{V}_{1}=z_{1}\left(z_{2}+e_{1}+\alpha_{1}+\lambda_{2}+f_{1}\left(\bar{x}_{1}, \omega_{1}(t)\right)+d_{1}(t)-\dot{y}_{d}\right) \\
-\frac{1}{\eta_{1}} \widetilde{\theta}_{1} \dot{\hat{\theta}}_{1}-\widetilde{W}_{1}^{T} \Gamma_{w_{1}}^{-1} \dot{\hat{W}}_{1}-\operatorname{tr}\left\{\widetilde{\gamma}_{1}^{T} \Gamma_{\gamma_{1}}^{-1} \dot{\hat{\gamma}}_{1}\right\}-\frac{1}{\beta_{1}} \tilde{N}_{1} \dot{\hat{N}}_{1} .
\end{gathered}
$$

$$
z_{1}\left[f_{1}\left(\bar{x}_{1}, \omega_{1}(t)\right)-\dot{y}_{d}+\lambda_{2}\right] \leq \frac{z_{1}^{2}\left[f_{1}\left(\bar{x}_{1}, \omega_{1}(t)\right)-\dot{y}_{d}+\lambda_{2}\right]^{2}}{\sqrt{z_{1}^{2}\left[f_{1}\left(\bar{x}_{1}, \omega_{1}(t)\right)-\dot{y}_{d}+\lambda_{2}\right]^{2}+\mu^{2}}}+\mu .
$$

Let $\quad g_{1}\left(E_{1}, \omega_{1}(t)\right)=\left(z_{1}\left[f_{1}\left(\bar{x}_{1}, \omega_{1}(t)\right)-\dot{y}_{d}+\lambda_{2}\right]^{2} /\right.$ $\left.\sqrt{z_{1}^{2}\left[f_{1}\left(\bar{x}_{1}, \omega_{1}(t)\right)-\dot{y}_{d}+\lambda_{2}\right]^{2}+\mu^{2}}\right)$, according to $(2)$ and Lemma 1 , it can be concluded that

$$
\begin{aligned}
g_{1}\left(E_{1}, \omega_{1}(t)\right) & =W_{1}^{T} S_{1}\left(E_{1}, \gamma_{1}^{T} \varphi_{1}(t)\right)+\delta_{1}\left(E_{1}, \omega_{1}(t)\right), \\
\left|\delta_{1}\left(E_{1}, \omega_{1}(t)\right)\right| & \leq \varepsilon_{1},
\end{aligned}
$$

$$
\begin{gathered}
\dot{V}_{1} \leq z_{1}\left(z_{2}+e_{1}+\alpha_{1}+\lambda_{2}+W_{1}^{T} S_{1}\left(E_{1}, \gamma_{1}^{T} \varphi_{1}(t)\right) z_{1}+\varepsilon_{1} z_{1}+d_{1}(t)\right) \\
-\frac{1}{\eta_{1}} \widetilde{\theta}_{1} \dot{\hat{\theta}}_{1}-\widetilde{W}_{1}^{T} \Gamma_{w_{1}}^{-1} \dot{\hat{W}}_{1}-\operatorname{tr}\left\{\widetilde{\gamma}_{1}^{T} \Gamma_{\gamma_{1}}^{-1} \dot{\hat{\gamma}}_{1}\right\}-\frac{1}{\beta_{1}} \widetilde{N}_{1} \dot{\hat{N}}_{1}+\mu
\end{gathered}
$$

where $E_{1}=\left[x_{1}, \dot{y}_{d}\right], \varepsilon_{1}$ denotes the upper bound of $\delta_{1}\left(E_{1}, \omega_{1}(t)\right)$. Substituting (16) and (17) into (15), we can get
Using Young's inequality, one has

$$
\begin{aligned}
& z_{1} e_{1} \leq \frac{1}{2} z_{1}^{2}+\frac{1}{2} e_{1}^{2}, \\
& z_{1} z_{2} \leq \frac{1}{2} z_{1}^{2}+\frac{1}{2} z_{2}^{2} .
\end{aligned}
$$

$$
\bar{\alpha}_{1}=-\left(k_{1}+\frac{3}{2}\right) z_{1}-\widehat{W}_{1}^{T} S_{1}\left(E_{1}, \widehat{\gamma}_{1}^{T} \varphi_{1}(t)\right) z_{1}-\widehat{\theta}_{1} \frac{z_{1}^{3} v_{1}^{2}}{\sqrt{z_{1}^{4} v_{1}^{2}+\mu^{2}}}-\frac{z_{1} \widehat{N}_{1}^{2}}{\sqrt{z_{1}^{2} \widehat{N}_{1}^{2}+\mu^{2}}}
$$

where the definition of $v_{1}$ will give in (24), $k_{1}$ is a positive parameter. Then, substituting (19), (20), and (21) into (18), we can get the following inequality:

$$
\begin{aligned}
\dot{V}_{1} \leq z_{1}\left[-k_{1} z_{1}+W_{1}^{T} S_{1}\left(E_{1}, \gamma_{1}^{T} \varphi_{1}(t)\right) z_{1}-\widehat{W}_{1}^{T} S_{1}\left(E_{1}, \widehat{\gamma}_{1}^{T} \varphi_{1}(t)\right) z_{1}+\varepsilon_{1} z_{1}-\widehat{\theta}_{1} \frac{z_{1}^{3} v_{1}^{2}}{\sqrt{z_{1}^{4} v_{1}^{2}+\mu^{2}}}\right. \\
\left.-\frac{z_{1} \widehat{N}_{1}^{2}}{\sqrt{z_{1}^{2} \widehat{N}_{1}^{2}+\mu^{2}}}+d_{1}(t)\right]-\frac{1}{\eta_{1}} \tilde{\theta}_{1} \dot{\hat{\theta}}_{1}-\widetilde{W}_{1}^{T} \Gamma_{w_{1}}^{-1} \dot{\hat{W}}_{1}-\operatorname{tr}\left\{\tilde{\gamma}_{1}^{T} \Gamma_{\gamma_{1}}^{-1} \dot{\hat{\gamma}}_{1}\right\}-\frac{1}{\beta_{1}} \tilde{N}_{1} \dot{\hat{N}}_{1}+\mu+\frac{1}{2} e_{1}^{2}+\frac{1}{2} z_{2}^{2}+z_{1} L_{\mathrm{st}}\left(\Phi_{1}\right) \bar{\alpha}_{1}-z_{1} \bar{\alpha}_{1} .
\end{aligned}
$$


According to Lemma 2, we have

$$
\begin{aligned}
\dot{V}_{1} \leq & z_{1}\left[-k_{1} z_{1}+\widetilde{W}_{1}^{T}\left(\widehat{S}_{1}-\widehat{S}_{1}^{\prime} \widehat{\gamma}_{1}^{T} \varphi_{1}(t)\right) z_{1}+\widehat{W}_{1}^{T} \widehat{S}_{1}^{\prime} \widetilde{\gamma}_{1}^{T} \varphi_{1}(t) z_{1}+b_{1} z_{1}+\varepsilon_{1} z_{1}-\widehat{\theta}_{1} \frac{z_{1}^{3} v_{1}^{2}}{\sqrt{z_{1}^{4} v_{1}^{2}+\mu^{2}}}\right. \\
& \left.-\frac{z_{1} \widehat{N}_{1}^{2}}{\sqrt{z_{1}^{2} \widehat{N}_{1}^{2}+\mu^{2}}}+d_{1}(t)\right]-\frac{1}{\eta_{1}} \widetilde{\theta}_{1} \dot{\hat{\theta}}_{1}-\widetilde{W}_{1}^{T} \Gamma_{w_{1}}^{-1} \dot{\hat{W}}_{1}-\operatorname{tr}\left\{\widetilde{\gamma}_{1}^{T} \Gamma_{\gamma_{1}}^{-1} \dot{\hat{\gamma}}_{1}\right\}-\frac{1}{\beta_{1}} \widetilde{N}_{1} \dot{\hat{\hat{N}}}_{1}+\mu+\frac{1}{2} e_{1}^{2}+\frac{1}{2} z_{2}^{2}+z_{1} L_{\mathrm{st}}\left(\bigotimes_{1}\right) \bar{\alpha}_{1}-z_{1} \bar{\alpha}_{1} .
\end{aligned}
$$

By using Lemmas 2 and 3, we can get

$$
\begin{aligned}
\left(b_{1}+\varepsilon_{1}\right) z_{1}^{2} \leq & {\left[\left\|\gamma_{1}\right\|_{F}\left\|\varphi_{1}(t) \widehat{W}_{1}^{T} \widehat{S}_{1}^{\prime}\right\|_{F}+\left\|W_{1}\right\|\left\|\varphi_{1}(t) \widehat{\gamma}_{1}^{T} \widehat{S}_{1}^{\prime}\right\|\right.} \\
& \left.+\left|W_{1}\right|_{1}+\left|\varepsilon_{1}\right|\right] z_{1}^{2} \\
\leq & \theta_{1}\left|z_{1}^{2} v_{1}\right| \\
\leq & \theta_{1} \frac{z_{1}^{4} v_{1}^{2}}{\sqrt{z_{1}^{4} v_{1}^{2}+\mu^{2}}}+\theta_{1} \mu
\end{aligned}
$$

$$
\begin{aligned}
z_{1} d_{1}(t) & \leq\left|z_{1}\right|\left|d_{1}(t)\right| \\
& \leq\left|z_{1}\right| N_{1}=\left|z_{1}\right| \widehat{N}_{1}+\left|z_{1}\right| \widetilde{N}_{1} \\
& \leq \frac{z_{1}^{2} \widehat{N}_{1}^{2}}{\sqrt{z_{1}^{2} \widehat{N}_{1}^{2}+\mu^{2}}}+\mu+\left|z_{1}\right| \widetilde{N}_{1},
\end{aligned}
$$

where

$$
\begin{aligned}
& \theta_{1}=\sqrt{\left\|\gamma_{1}\right\|_{F}^{2}+\left\|W_{1}\right\|^{2}+\left(\left|W_{1}\right|_{1}+\left|\varepsilon_{1}\right|\right)^{2}}, \\
& v_{1}=\sqrt{\left\|\varphi_{1}(t) \widehat{W}_{1}^{T} \widehat{S}_{1}^{\prime}\right\|_{F}^{2}+\left\|\varphi_{1}(t) \widehat{\gamma}_{1}^{T} \widehat{S}_{1}^{\prime}\right\|^{2}+1} .
\end{aligned}
$$

Combining (23)-(25), the following inequality holds:

$$
\begin{aligned}
\dot{V}_{1} \leq & z_{1}\left[-k_{1} z_{1}+\widetilde{W}_{1}^{T}\left(\widehat{S}_{1}-\widehat{S}_{1}^{\prime} \widehat{\gamma}_{1}^{T} \varphi_{1}(t)\right) z_{1}+\widehat{W}_{1}^{T} \widehat{S}_{1}^{\prime} \widetilde{\gamma}_{1}^{T} \varphi_{1}(t) z_{1}-\widetilde{\theta}_{1} \frac{z_{1}^{3} v_{1}^{2}}{\sqrt{z_{1}^{4} v_{1}^{2}+\mu^{2}}}\right] \\
& +\left(\theta_{1}+2\right) \mu+\left|z_{1}\right| \widetilde{N}_{1}-\frac{1}{\eta_{1}} \tilde{\theta}_{1} \dot{\hat{\theta}}_{1}-\widetilde{W}_{1}^{T} \Gamma_{w_{1}}^{-1} \dot{\hat{W}}_{1}-\operatorname{tr}\left\{\widetilde{\gamma}_{1}^{T} \Gamma_{\gamma_{1}}^{-1} \dot{\hat{\gamma}}_{1}\right\}-\frac{1}{\beta_{1}} \widetilde{N}_{1} \dot{\hat{N}}_{1} \\
& +\frac{1}{2} e_{1}^{2}+\frac{1}{2} z_{2}^{2}+z_{1} L_{\text {st }}\left(\oplus_{1}\right) \bar{\alpha}_{1}-z_{1} \bar{\alpha}_{1},
\end{aligned}
$$

where $\widehat{W}_{1}^{T} \widehat{S}_{1}^{\prime} \widetilde{\gamma}_{1}^{T} \varphi_{1}(t)=\operatorname{tr}\left\{\widehat{W}_{1}^{T} \widehat{S}_{1}^{\prime} \widetilde{\gamma}_{1}^{T} \varphi_{1}(t)\right\}$. Then, one
has

$$
\begin{aligned}
\dot{V}_{1} \leq & -k_{1} z_{1}^{2}+\frac{1}{2} e_{1}^{2}+\frac{1}{2} z_{2}^{2}+\left(\theta_{1}+2\right) \mu+z_{1} L_{\mathrm{st}}\left(\varpi_{1}\right) \bar{\alpha}_{1}-z_{1} \bar{\alpha}_{1}+\widetilde{W}_{1}^{T}\left[z_{1}^{2}\left(\widehat{S}_{1}-\widehat{S}_{1}^{\prime} \hat{\widehat{\gamma}}_{1}^{T} \varphi_{1}(t)\right)-\Gamma_{w_{1}}^{-1} \dot{\hat{W}}_{1}\right] \\
& +\widetilde{\theta}_{1}\left(\frac{z_{1}^{4} v_{1}^{2}}{\sqrt{z_{1}^{4} v_{1}^{2}+\mu^{2}}}-\frac{1}{\eta_{1}} \dot{\hat{\theta}}_{1}\right)+\widetilde{N}_{1}\left(\left|z_{1}\right|-\frac{1}{\beta_{1}} \dot{\hat{N}}_{1}\right)+\operatorname{tr}\left\{\widetilde{\gamma}_{1}^{T}\left(\widehat{W}_{1}^{T} \widehat{S}_{1}^{\prime} \varphi_{1}(t) z_{1}^{2}-\Gamma_{\gamma_{1}}^{-1} \dot{\hat{\gamma}}_{1}\right)\right\} .
\end{aligned}
$$


Based on (28), the adaptive laws are constructed as follows:

$$
\left\{\begin{array}{l}
\dot{\hat{\theta}}_{1}=\eta_{1} \frac{z_{1}^{4} v_{1}^{2}}{\sqrt{z_{1}^{4} v_{1}^{2}+\mu^{2}}}-\rho_{1} \widehat{\theta}_{1} \\
\dot{\hat{W}}_{1}=\Gamma_{W_{1}}\left[z_{1}^{2}\left(\widehat{S}_{1}-\widehat{S}_{1}^{\prime} \widehat{\gamma}_{1}^{T} \varphi_{1}(t)\right)-\sigma_{1} \widehat{W}_{1}\right] \\
\dot{\hat{\gamma}}_{1}=\Gamma_{\gamma_{1}}\left[z_{1}^{2} \widehat{W}_{1}^{T} \widehat{S}_{1}^{\prime} \varphi_{1}(t)-\kappa_{1} \widehat{\gamma}_{1}\right] \\
\dot{\hat{N}}_{1}=\beta_{1}\left|z_{1}\right|-o_{1} \widehat{N}_{1}, \\
\dot{\hat{\omega}}_{1}=-D_{1} z_{1} \bar{\alpha}_{1},
\end{array}\right.
$$

where $\rho_{1}, \sigma_{1}, \kappa_{1}, o_{1}$, and $D_{1}$ are positive design parameters. Then, substituting (29) into (28) produces

$$
\begin{aligned}
\dot{V}_{1} \leq & -k_{1} z_{1}^{2}+\frac{1}{2} e_{1}^{2}+\frac{1}{2} z_{2}^{2}+\left(\theta_{1}+2\right) \mu+\frac{\rho_{1}}{\eta_{1}} \widetilde{\theta}_{1} \widehat{\theta}_{1}+\sigma_{1} \widetilde{W}_{1}^{T} \widehat{W}_{1}+\kappa_{1} \operatorname{tr}\left\{\widetilde{\gamma}_{1}^{T} \widehat{\gamma}_{1}\right\} \\
& +\frac{o_{1}}{\beta_{1}} \widetilde{N}_{1} \widehat{N}_{1}+\frac{1}{D_{1}} \dot{\omega}_{1}(t)-\frac{1}{D_{1}} L_{\text {st }}\left(\Phi_{1}(t)\right) \dot{\omega}_{1}(t) .
\end{aligned}
$$

Using Young's inequality, the following inequalities Therefore, substituting (31) into (30), we have hold:

$$
\begin{aligned}
\dot{V}_{1} \leq & -k_{1} z_{1}^{2}+\frac{1}{2} e_{1}^{2}+\frac{1}{2} z_{2}^{2}-\frac{\rho_{1}}{2 \eta_{1}} \tilde{\theta}_{1}^{2}-\frac{\sigma_{1}}{2}\left\|\tilde{W}_{1}\right\|^{2}-\frac{\kappa_{1}}{2}\left\|\widetilde{\gamma}_{1}\right\|_{F}^{2}-\frac{o_{1}}{2 \beta_{1}} \tilde{N}_{1}^{2} \\
& +A_{1}+\frac{1}{D_{1}} \dot{\oplus}_{1}(t)-\frac{1}{D_{1}} L_{\text {st }}\left(\Phi_{1}(t)\right) \dot{\omega}_{1}(t),
\end{aligned}
$$

where

$A_{1}=\left(\theta_{1}+2\right) \mu+\frac{\rho_{1}}{2 \eta_{1}} \theta_{1}^{2}+\frac{\sigma_{1}}{2}\left\|W_{1}\right\|^{2}+\frac{\kappa_{1}}{2}\left\|\gamma_{1}\right\|_{F}^{2}+\frac{o_{1}}{2 \beta_{1}} N_{1}^{2}$.

\footnotetext{
Step 2: similar to (13), then the derivative of $z_{i}$ is
}

$$
\begin{aligned}
\dot{z}_{i} & =\dot{x}_{i}-\dot{s}_{i-1}-\dot{\lambda}_{i} \\
& =x_{i+1}+f_{i}\left(\bar{x}_{i}, \omega_{i}(t)\right)+d_{i}(t)-\dot{s}_{i-1}-\dot{\lambda}_{i+1}+p_{i} \lambda_{i} \\
& =z_{i+1}+s_{i}+f_{i}\left(\bar{x}_{i}, \omega_{i}(t)\right)+d_{i}(t)-\dot{s}_{i-1}+p_{i} \lambda_{i} \\
& =z_{i+1}+e_{i}+\alpha_{i}+f_{i}\left(\bar{x}_{i}, \omega_{i}(t)\right)+d_{i}(t)-\dot{s}_{i-1}+p_{i} \lambda_{i} .
\end{aligned}
$$


The Lyapunov function $V_{i}$ is constructed

$$
\begin{aligned}
V_{i}= & V_{i-1}+\frac{1}{2} z_{i}^{2}+\frac{1}{2 \eta_{i}} \tilde{\theta}_{i}^{2}+\frac{1}{2} \tilde{W}_{i}^{T} \Gamma_{w_{i}}^{-1} \tilde{W}_{i}+\frac{1}{2} \operatorname{tr}\left\{\tilde{\gamma}_{i}^{T} \Gamma_{\gamma_{i}}^{-1} \tilde{\gamma}_{i}\right\} \\
& +\frac{1}{2 \beta_{i}} \tilde{N}_{i}^{2},
\end{aligned}
$$

where $\eta_{i}>0$ and $\beta_{i}>0$ are the design parameters, the estimation errors $\theta_{i}=\theta_{i}-\widehat{\theta}_{i}, \widetilde{N}_{i}=N_{i}-\widehat{N}_{i}, \widehat{\theta}_{i}$ and $\widehat{N}_{i}$ represent the estimation of $\theta_{i}$ and $N_{i}$, respectively. $\Gamma_{w_{i}}>0$ and $\Gamma_{\gamma_{i}}>0$ are the invertible matrices of the design. Similar to (15), one has

$$
\begin{aligned}
\dot{V}_{i}= & \dot{V}_{i-1}+z_{i}\left(z_{i+1}+e_{i}+\alpha_{i}+f_{i}\left(\bar{x}_{i}, \omega_{i}(t)\right)+d_{i}(t)-\dot{s}_{i-1}+p_{i} \lambda_{i}\right) \\
& -\frac{1}{\eta_{i}} \tilde{\theta}_{i} \dot{\hat{\theta}}_{i}-\widetilde{W}_{i}^{T} \Gamma_{w_{i}}^{-1} \dot{\hat{W}}_{i}-\operatorname{tr}\left\{\widetilde{\gamma}_{i}^{T} \Gamma_{\gamma_{i}}^{-1} \dot{\hat{\gamma}}_{i}\right\}-\frac{1}{\beta_{i}} \widetilde{N}_{i} \dot{\hat{N}}_{i} .
\end{aligned}
$$

Similar to (16), the following inequality can be obtained:

$$
z_{i}\left[f_{i}\left(\bar{x}_{i}, \omega_{i}(t)\right)-\dot{s}_{i-1}+p_{i} \lambda_{i}\right] \leq \frac{z_{i}^{2}\left[f_{i}\left(\bar{x}_{i}, \omega_{i}(t)\right)-\dot{s}_{i-1}+p_{i} \lambda_{i}\right]^{2}}{\sqrt{z_{i}^{2}\left[f_{i}\left(\bar{x}_{i}, \omega_{i}(t)\right)-\dot{s}_{i-1}+p_{i} \lambda_{i}\right]^{2}+\mu^{2}}}+\mu
$$

Let

$$
\begin{aligned}
g_{i}\left(E_{i}, \omega_{i}(t)\right) & =W_{i}^{T} S_{i}\left(E_{i}, \gamma_{i}^{T} \varphi_{i}(t)\right)+\delta_{i}\left(E_{i}, \omega_{i}(t)\right), \\
\left|\delta_{i}\left(E_{i}, \omega_{i}(t)\right)\right| & \leq \varepsilon_{i},
\end{aligned}
$$

$g_{i}\left(E_{i}, \omega_{i}(t)\right)=\frac{z_{i}\left[f_{i}\left(\bar{x}_{i}, \omega_{i}(t)\right)-\dot{s}_{i-1}+p_{i} \lambda_{i}\right]^{2}}{\sqrt{z_{i}^{2}\left[f_{i}\left(\bar{x}_{i}, \omega_{i}(t)\right)-\dot{s}_{i-1}+p_{i} \lambda_{i}\right]^{2}+\mu^{2}}}$,

then according to (2) and Lemma 1, we can get the where $E_{i}=\left[x_{1}, \ldots, x_{i}, \dot{s}_{1}, \ldots, \dot{s}_{i-1}, \lambda_{2}, \ldots, \lambda_{i}\right]$ and $\varepsilon_{i}$ denotes the upper bound of $\delta_{i}\left(E_{i}, \omega_{i}(t)\right)$. Then, substituting (37) and (39) into (36), one has

following conclusions:

$$
\begin{gathered}
\dot{V}_{i} \leq \dot{V}_{i-1}+z_{i}\left(z_{i+1}+e_{i}+\alpha_{i}+W_{i}^{T} S_{i}\left(E_{i}, \gamma_{i}^{T} \varphi_{i}(t)\right) z_{i}+\varepsilon_{i} z_{i}+d_{i}(t)\right) \\
-\frac{1}{\eta_{i}} \tilde{\theta}_{i} \dot{\hat{\theta}}_{i}-\widetilde{W}_{i}^{T} \Gamma_{w_{i}}^{-1} \dot{\hat{W}}_{i}-\operatorname{tr}\left\{\widetilde{\gamma}_{i}^{T} \Gamma_{\gamma_{i}}^{-1} \dot{\hat{\gamma}}_{i}\right\}-\frac{1}{\beta_{i}} \widetilde{N}_{i} \dot{\hat{N}}_{i}+\mu
\end{gathered}
$$

Using Young's inequality, we have

$$
\begin{aligned}
z_{i} e_{i} & \leq \frac{1}{2} z_{i}^{2}+\frac{1}{2} e_{i}^{2}, \\
z_{i} z_{i+1} & \leq \frac{1}{2} z_{i}^{2}+\frac{1}{2} z_{i+1}^{2} .
\end{aligned}
$$

The design of virtual controller $\alpha_{i}$ is designed as follows:

$$
\alpha_{i}=L_{\mathrm{st}}\left(\varpi_{i}(t)\right) \bar{\alpha}_{i}
$$

where $L_{s t}(\cdot)$ is a dynamic gain function in Lemma 4 , the adaptive law of $\omega_{i}$ is given in (51), and $\bar{\alpha}_{i}$ is the equivalent unit of virtual controller $\alpha_{i}$

$$
\bar{\alpha}_{i}=-\left(k_{i}+\frac{3}{2}\right) z_{i}-\widehat{W}_{i}^{T} S_{i}\left(E_{i}, \widehat{\gamma}_{i}^{T} \varphi_{i}(t)\right) z_{i}-\widehat{\theta}_{i} \frac{z_{i}^{3} v_{i}^{2}}{\sqrt{z_{i}^{4} v_{i}^{2}+\mu^{2}}}-\frac{z_{i} \widehat{N}_{i}^{2}}{\sqrt{z_{i}^{2} \widehat{N}_{i}^{2}+\mu^{2}}}
$$


where the definition of $v_{i}$ will give in formula (46), $k_{i}$ is the positive parameter of the design. Substituting (41)-(43) into (40), the following inequality holds:

$$
\begin{aligned}
\dot{V}_{i} \leq & \dot{V}_{i-1}+z_{i}\left[\left(-k_{i}+\frac{1}{2}\right) z_{i}+W_{i}^{T} S_{i}\left(E_{i}, \gamma_{i}^{T} \varphi_{i}(t)\right) z_{i}-\widehat{W}_{i}^{T} S_{i}\left(E_{i}, \widehat{\gamma}_{i}^{T} \varphi_{i}(t)\right) z_{i}+\varepsilon_{i} z_{i}-\widehat{\theta}_{i} \frac{z_{i}^{3} v_{i}^{2}}{\sqrt{z_{i}^{4} v_{i}^{2}+\mu^{2}}}\right. \\
& \left.-\frac{z_{i} \widehat{N}_{i}^{2}}{\sqrt{z_{i}^{2} \widehat{N}_{i}^{2}+\mu^{2}}}+d_{i}(t)\right]-\frac{1}{\eta_{i}} \tilde{\theta}_{i} \dot{\hat{\theta}}_{i}-\widetilde{W}_{i}^{T} \Gamma_{w_{i}}^{-1} \dot{\hat{W}}_{i}-\operatorname{tr}\left\{\tilde{\gamma}_{i}^{T} \Gamma_{\gamma_{i}}^{-1} \dot{\hat{\gamma}}_{i}\right\}-\frac{1}{\beta_{i}} \widetilde{N}_{i} \dot{\hat{N}}_{i}+\mu+\frac{1}{2} e_{i}^{2}+\frac{1}{2} z_{i+1}^{2}+z_{i} L_{\mathrm{st}}\left(\varrho_{i}\right) \bar{\alpha}_{i}-z_{i} \bar{\alpha}_{i} .
\end{aligned}
$$

According to Lemma 2, we have

$$
\begin{aligned}
\dot{V}_{i} \leq & \dot{V}_{i-1}+z_{i}\left[\left(-k_{i}+\frac{1}{2}\right) z_{i}+\widetilde{W}_{i}^{T}\left(\widehat{S}_{i}-\widehat{S}_{i}^{\prime} \widehat{\gamma}_{i}^{T} \varphi_{i}(t)\right) z_{i}-\widehat{W}_{i}^{T} \widehat{S}_{i}^{\prime} \widetilde{\gamma}_{i}^{T} \varphi_{i}(t) z_{i}+b_{i} z_{i}+\varepsilon_{i} z_{i}-\widehat{\theta}_{i} \frac{z_{i}^{3} v_{i}^{2}}{\sqrt{z_{i}^{4} v_{i}^{2}+\mu^{2}}}\right. \\
& \left.-\frac{z_{i} \widehat{N}_{i}^{2}}{\sqrt{z_{i}^{2} \widehat{N}_{i}^{2}+\mu^{2}}}+d_{i}(t)\right]-\frac{1}{\eta_{i}} \tilde{\theta}_{i} \dot{\hat{\theta}}_{i}-\widetilde{W}_{i}^{T} \Gamma_{w_{i}}^{-1} \dot{\hat{W}}_{i}-\operatorname{tr}\left\{\widetilde{\gamma}_{i}^{T} \Gamma_{\gamma_{i}}^{-1} \hat{\hat{\gamma}}_{i}\right\}-\frac{1}{\beta_{i}} \widetilde{N}_{i} \dot{\hat{N}}_{i}+\mu+\frac{1}{2} e_{i}^{2}+\frac{1}{2} z_{i+1}^{2}+z_{i} L_{\mathrm{st}}\left(\bigotimes_{i}\right) \bar{\alpha}_{i}-z_{i} \bar{\alpha}_{i} .
\end{aligned}
$$

By using Lemmas 2 and 3, we can get

$$
\begin{aligned}
&\left.+\left|W_{i}\right|_{1}+\left|\varepsilon_{i}\right|\right] z_{i}^{2} \\
& \leq \theta_{i}\left|z_{i}^{2} v_{i}\right| \\
& \leq \theta_{i} \frac{z_{i}^{4} v_{i}^{2}}{\sqrt{z_{i}^{4} v_{i}^{2}+\mu^{2}}}+\theta_{i} \mu, \\
& z_{i} d_{i}(t) \leq\left|z_{i}\right|\left|d_{i}(t)\right| \\
& \leq\left|z_{i}\right| N_{i}=\left|z_{i}\right| \widehat{N}_{i}+\left|z_{i}\right| \widetilde{N}_{i} \\
& \leq \frac{z_{i}^{2} \widehat{N}_{i}^{2}}{\sqrt{z_{i}^{2} \widehat{N}_{i}^{2}+\mu^{2}}}+\mu+\left|z_{i}\right| \widetilde{N}_{i},
\end{aligned}
$$$$
\left(b_{i}+\varepsilon_{i}\right) z_{i}^{2} \leq\left[\left\|\gamma_{i}\right\|_{F}\left\|\varphi_{i}(t) \widehat{W}_{i}^{T} \widehat{S}_{i}^{\prime}\right\|_{F}+\left\|W_{i}\right\|\left\|\varphi_{i}(t) \widehat{\gamma}_{i}^{T} \widehat{S}_{i}^{\prime}\right\|\right.
$$

where

$$
\begin{aligned}
& \theta_{i}=\sqrt{\left\|\gamma_{i}\right\|_{F}^{2}+\left\|W_{i}\right\|^{2}+\left(\left|W_{i}\right|_{1}+\left|\varepsilon_{i}\right|\right)^{2}}, \\
& v_{i}=\sqrt{\left\|\varphi_{i}(t) \widehat{W}_{i}^{T} \hat{S}_{i}^{\prime}\right\|_{F}^{2}+\left\|\varphi_{i}(t) \widehat{\gamma}_{i}^{T} \widehat{S}_{i}^{\prime}\right\|^{2}+1} .
\end{aligned}
$$

Substituting (46) and (47) into (45), the following inequality holds:

$$
\begin{aligned}
\dot{V}_{i} \leq & \dot{V}_{i-1}+z_{i}\left[-\left(k_{i}+\frac{1}{2}\right) z_{i}+\widetilde{W}_{i}^{T}\left(\widehat{S}_{i}-\widehat{S}_{i}^{\prime} \widehat{\gamma}_{i}^{T} \varphi_{i}(t)\right) z_{i}+\widehat{W}_{i}^{T} \widehat{S}_{i} \widetilde{\gamma}_{i}^{T} \varphi_{i}(t) z_{i}-\widetilde{\theta}_{i} \frac{z_{i}^{3} v_{i}^{2}}{\sqrt{z_{i}^{4} v_{i}^{2}+\mu^{2}}}\right] \\
& +\left(\theta_{i}+2\right) \mu+\left|z_{i}\right| \widetilde{N}_{i}-\frac{1}{\eta_{i}} \tilde{\theta}_{i} \dot{\hat{\theta}}_{i}-\widetilde{W}_{i}^{T} \Gamma_{w_{i}}^{-1} \dot{\hat{W}}_{i}-\operatorname{tr}\left\{\widetilde{\gamma}_{i}^{T} \Gamma_{\gamma_{i}}^{-1} \dot{\hat{\gamma}}_{i}\right\}-\frac{1}{\beta_{i}} \widetilde{N}_{i} \dot{\hat{\hat{N}}}_{i} \\
& +\frac{1}{2} e_{i}^{2}+\frac{1}{2} z_{i+1}^{2}+z_{i} L_{\mathrm{st}}\left(\oplus_{i}\right) \bar{\alpha}_{i}-z_{i} \bar{\alpha}_{i},
\end{aligned}
$$


where $\widehat{W}_{i}^{T} \widehat{S}_{i}^{\prime} \widetilde{\gamma}_{i}^{T} \varphi_{i}(t)=\operatorname{tr}\left\{\widehat{W}_{i}^{T} \widehat{S}_{i}^{\prime} \widetilde{\gamma}_{i}^{T} \varphi_{i}(t)\right\}$. Then, one has

$$
\begin{aligned}
\dot{V}_{i} \leq & \dot{V}_{i-1}-\left(k_{i}+\frac{1}{2}\right) z_{i}^{2}+\frac{1}{2} e_{i}^{2}+\frac{1}{2} z_{i+1}^{2}+\left(\theta_{i}+2\right) \mu+z_{i} L_{\mathrm{st}}\left(\bigotimes_{i}\right) \bar{\alpha}_{i}-z_{i} \bar{\alpha}_{i}+\tilde{W}_{i}^{T}\left[z_{i}^{2}\left(\widehat{S}_{i}-\widehat{S}_{i}^{\prime} \widehat{\gamma}_{i}^{T} \varphi_{i}(t)\right)\right. \\
& \left.-\Gamma_{w_{i}}^{-1} \dot{\hat{W}}_{i}\right]+\tilde{\theta}_{i}\left(\frac{z_{i}^{4} v_{i}^{2}}{\sqrt{z_{i}^{4} v_{i}^{2}+\mu^{2}}}-\frac{1}{\eta_{i}} \dot{\hat{\theta}}_{i}\right)+\tilde{N}_{i}\left(\left|z_{i}\right|-\frac{1}{\beta_{i}} \dot{\hat{N}}_{i}\right)
\end{aligned}
$$

Based on (50), the adaptive laws are constructed as follows:

$$
\left\{\begin{array}{l}
\dot{\hat{\theta}}_{i}=\eta_{i} \frac{z_{i}^{4} v_{i}^{2}}{\sqrt{z_{i}^{4} v_{i}^{2}+\mu^{2}}}-\rho_{i} \widehat{\theta}_{i}, \\
\dot{\hat{W}}_{i}=\Gamma_{W_{i}}\left[z_{i}^{2}\left(\widehat{S}_{i}-\widehat{S}_{i}^{\prime} \widehat{\gamma}_{i}^{T} \varphi_{i}(t)\right)-\sigma_{i} \widehat{W}_{i}\right], \\
\dot{\hat{\gamma}}_{i}=\Gamma_{\gamma_{i}}\left[z_{i}^{2} \widehat{W}_{i}^{T} \widehat{S}_{i}^{\prime} \varphi_{i}(t)-\kappa_{i} \widehat{\gamma}_{i}\right], \\
\dot{\hat{N}}_{i}=\beta_{i}\left|z_{i}\right|-o_{i} \widehat{N}_{i}, \\
\dot{\omega}_{i}=-D_{i} z_{i} \bar{\alpha}_{i},
\end{array}\right.
$$

$$
\begin{aligned}
\dot{V}_{i} \leq & \dot{V}_{i-1}-\left(k_{i}+\frac{1}{2}\right) z_{i}^{2}+\frac{1}{2} e_{i}^{2}+\frac{1}{2} z_{i+1}^{2}+\left(\theta_{i}+2\right) \mu+\frac{\rho_{i}}{\eta_{i}} \widetilde{\theta}_{i} \widehat{\theta}_{i}+\sigma_{i} \widetilde{W}_{i}^{T} \widehat{W}_{i}+\kappa_{i} \operatorname{tr}\left\{\widetilde{\gamma}_{i}^{T} \widehat{\gamma}_{i}\right\} \\
& +\frac{o_{i}}{\beta_{i}} \widetilde{N}_{i} \widehat{N}_{i}+\frac{1}{D_{i}} \dot{\oplus}_{i}(t)-\frac{1}{D_{i}} L_{\mathrm{st}}\left(\oplus_{i}(t)\right) \dot{\oplus}_{i}(t) .
\end{aligned}
$$

Using Young's inequality, the following inequalities hold:

$$
\left\{\begin{array}{l}
\frac{\rho_{i}}{\eta_{i}} \widetilde{\theta}_{i} \widehat{\theta}_{i} \leq-\frac{\rho_{i}}{2 \eta_{i}} \widetilde{\theta}_{i}^{2}+\frac{\rho_{i}}{2 \eta_{i}} \theta_{i}^{2}, \\
\sigma_{i} \widetilde{W}_{i} \widehat{W}_{i}^{T} \leq-\frac{\sigma_{i}}{2}\left\|\widetilde{W}_{i}\right\|^{2}+\frac{\sigma_{i}}{2}\left\|W_{i}\right\|^{2}, \\
\kappa_{i} \operatorname{tr}\left\{\widetilde{\gamma}_{i}^{T} \widehat{\gamma}_{i}\right\} \leq-\frac{\kappa_{i}}{2}\left\|\widetilde{\gamma}_{i}\right\|_{F}^{2}+\frac{\kappa_{i}}{2}\left\|\gamma_{i}\right\|_{F}^{2}, \\
\frac{o_{i}}{\beta_{i}} \widetilde{N}_{i} \widehat{N}_{i} \leq-\frac{o_{i}}{2 \beta_{i}} \widetilde{N}_{i}^{2}+\frac{o_{i}}{2 \beta_{i}} N_{i}^{2},
\end{array}\right.
$$

$$
\begin{aligned}
\dot{V}_{i} \leq & -\sum_{j=1}^{i} k_{j} z_{j}^{2}+\sum_{j=1}^{i} \frac{1}{2} e_{j}^{2}+\frac{1}{2} z_{i+1}^{2}-\sum_{j=1}^{i} \frac{\rho_{j}}{2 \eta_{j}} \widetilde{\theta}_{j}^{2}-\sum_{j=1}^{i} \frac{\sigma_{j}}{2}\left\|\tilde{W}_{j}\right\|^{2}-\sum_{j=1}^{i} \frac{\kappa_{j}}{2}\left\|\widetilde{\gamma}_{j}\right\|_{F}^{2}-\sum_{j=1}^{i} \frac{o_{j}}{2 \beta_{j}} \widetilde{N}_{j}^{2} \\
& +A_{i}+\sum_{j=1}^{i} \frac{1}{D_{j}} \dot{\boldsymbol{\omega}}_{j}(t)-\sum_{j=1}^{i} \frac{1}{D_{j}} L_{\mathrm{st}}\left({\omega_{j}}_{j}(t)\right) \dot{\boldsymbol{\omega}}_{j}(t),
\end{aligned}
$$

Substituting (53) into (52), we can get the following inequality: 
where

$$
\begin{aligned}
A_{i}= & \sum_{j=1}^{i}\left(\theta_{j}+2\right) \mu+\sum_{j=1}^{i} \frac{\rho_{j}}{2 \eta_{j}} \theta_{j}^{2}+\sum_{j=1}^{i} \frac{\sigma_{j}}{2}\left\|W_{j}\right\|^{2}+\sum_{j=1}^{i} \frac{\kappa_{j}}{2}\left\|\gamma_{j}\right\|_{F}^{2} \\
& +\sum_{j=1}^{i} \frac{o_{j}}{2 \beta_{j}} N_{j}^{2} .
\end{aligned}
$$

$$
\begin{aligned}
\dot{z}_{n} & =\dot{x}_{n}-\dot{s}_{n-1}-\dot{\lambda}_{n} \\
& =u(t-\tau(t))+f_{n}\left(\bar{x}_{n}, \omega_{n}(t)\right)+d_{n}(t)-\dot{s}_{n-1}+p_{n} \lambda_{n}-u(t-\tau(t))+u(t) \\
& =f_{n}\left(\bar{x}_{n}, \omega_{n}(t)\right)+d_{n}(t)-\dot{s}_{n-1}+p_{n} \lambda_{n}+u(t) .
\end{aligned}
$$

The Lyapunov function $V_{n}$ is constructed as follows:

$$
\begin{aligned}
V_{n}= & V_{n-1}+\frac{1}{2} z_{n}^{2}+\frac{1}{2 \eta_{n}} \widetilde{\theta}_{n}^{2}+\frac{1}{2} \widetilde{W}_{n}^{T} \Gamma_{w_{n}}^{-1} \widetilde{W}_{n}+\frac{1}{2} \operatorname{tr}\left\{\widetilde{\gamma}_{n}^{T} \Gamma_{\gamma_{n}}^{-1} \widetilde{\gamma}_{n}\right\} \\
& +\frac{1}{2 \beta_{n}} \widetilde{N}_{n}^{2},
\end{aligned}
$$

where $\eta_{n}>0$ and $\beta_{n}>0$ are the design parameters, the estimation errors $\widetilde{\theta}_{n}=\theta_{n}-\widehat{\theta}_{n}, \widetilde{N}_{n}=N_{n}-\widehat{N}_{n}, \widehat{\theta}_{n}$ and $\widehat{N}_{n}$ represent the estimation of $\theta_{n}$ and $N_{n}$, respectively. $\Gamma_{w_{n}}>0$ and $\Gamma_{\gamma_{n}}>0$ are the invertible matrices of the design. According to (57), it can be concluded that

$$
\begin{aligned}
\dot{V}_{n}= & \dot{V}_{n-1}+z_{n}\left(f_{n}\left(\bar{x}_{n}, \omega_{n}(t)\right)+d_{n}(t)-\dot{s}_{n-1}+p_{n} \lambda_{n}+u(t)\right) \\
& -\frac{1}{\eta_{n}} \tilde{\theta}_{n} \dot{\hat{\theta}}_{n}-\widetilde{W}_{n}^{T} \Gamma_{w_{n}}^{-1} \dot{\hat{W}}_{n}-\operatorname{tr}\left\{\widetilde{\gamma}_{n}^{T} \Gamma_{\gamma_{n}}^{-1} \dot{\hat{\gamma}}_{n}\right\}-\frac{1}{\beta_{n}} \widetilde{N}_{n} \dot{\hat{N}}_{n} .
\end{aligned}
$$

According to Lemma 3, one has

$$
\begin{aligned}
& \begin{array}{l}
z_{n}\left[f_{n}\left(\bar{x}_{n}, \omega_{n}(t)\right)-\dot{s}_{n-1}+p_{n} \lambda_{n}\right] \leq \\
z_{n}\left[f_{n}\left(\bar{x}_{n}, \omega_{n}(t)\right)-\dot{s}_{n-1}+p_{n} \lambda_{n}\right]^{2} \\
\left.z_{n}\left[\bar{x}_{n}, \omega_{n}(t)\right)-\dot{s}_{n-1}+p_{n} \lambda_{n}\right]^{2}+\mu^{2}
\end{array} \\
& \leq \frac{z_{n}^{2}\left[f_{n}\left(\bar{x}_{n}, \omega_{n}(t)\right)-\dot{s}_{n-1}+p_{n} \lambda_{n}\right]^{2}}{\sqrt{z_{n}^{2}\left[f_{n}\left(\bar{x}_{n}, \omega_{n}(t)\right)-\dot{s}_{n-1}+p_{n} \lambda_{n}\right]^{2}+\mu^{2}}}+\mu \\
& g_{n}\left(E_{n}, \omega_{n}(t)\right)=\frac{z_{n}\left[f_{n}\left(\bar{x}_{n}, \omega_{n}(t)\right)-\dot{s}_{n-1}+p_{n} \lambda_{n}\right]^{2}}{\sqrt{z_{n}^{2}\left[f_{n}\left(\bar{x}_{n}, \omega_{n}(t)\right)-\dot{s}_{n-1}+p_{n} \lambda_{n}\right]^{2}+\mu^{2}}} . \\
& g_{n}\left(E_{n}, \omega_{n}(t)\right)=W_{n}^{T} S_{n}\left(E_{n}, \gamma_{n}^{T} \varphi_{n}(t)\right)+\delta_{n}\left(E_{n}, \omega_{n}(t)\right) \text {, } \\
& \left|\delta_{n}\left(E_{n}, \omega_{n}(t)\right)\right| \leq \varepsilon_{n} \text {, }
\end{aligned}
$$

where $E_{n}=\left[x_{1}, \ldots, x_{n}, \dot{s}_{1}, \ldots, \dot{s}_{n-1}, \lambda_{2}, \ldots, \lambda_{n}\right], \varepsilon_{n}$ denotes the upper bound of $\delta_{n}\left(E_{n}, \omega_{n}(t)\right)$. Combining (58)-(61), the following inequality holds

$$
\dot{V}_{n} \leq \dot{V}_{n-1}+z_{n}\left(u+W_{n}^{T} S_{n}\left(E_{n}, \gamma_{n}^{T} \varphi_{n}(t)\right) z_{n}+\varepsilon_{n} z_{n}+d_{n}(t)\right)
$$

$$
-\frac{1}{\eta_{n}} \tilde{\theta}_{n} \dot{\hat{\theta}}_{n}-\widetilde{W}_{n}^{T} \Gamma_{w_{n}}^{-1} \dot{\hat{W}}_{n}-\operatorname{tr}\left\{\widetilde{\gamma}_{n}^{T} \Gamma_{\gamma_{n}}^{-1} \dot{\hat{\gamma}}_{n}\right\}-\frac{1}{\beta_{n}} \widetilde{N}_{n} \dot{\hat{N}}_{n}+\mu .
$$

The controller $u$ is designed as follows:

$$
u=L_{\mathrm{st}}\left(\oplus_{n}(t)\right) \bar{u},
$$

where $L_{\mathrm{st}}(\cdot)$ is a dynamic gain function in Lemma 4, the adaptive law of $\omega_{n}$ will give in (72), and $\bar{u}$ is the equivalent unit of virtual controller $u$ :

$$
\bar{u}=-\left(k_{n}+\frac{1}{2}\right) z_{n}-\widehat{W}_{n}^{T} S_{n}\left(E_{n}, \widehat{\gamma}_{n}^{T} \varphi_{n}(t)\right) z_{n}-\widehat{\theta}_{n} \frac{z_{n}^{3} v_{n}^{2}}{\sqrt{z_{n}^{4} v_{n}^{2}+\mu^{2}}}-\frac{z_{n} \widehat{N}_{n}^{2}}{\sqrt{z_{n}^{2} \widehat{N}_{n}^{2}+\mu^{2}}}
$$


where the definition of $v_{n}$ will give in (67), $k_{n}$ is the positive parameter of the design.
Similar to (44), the following inequality holds

$$
\begin{aligned}
\dot{V}_{n} \leq & \dot{V}_{n-1}+z_{n}\left[\left(-k_{n}+\frac{1}{2}\right) z_{n}+W_{n}^{T} S_{n}\left(E_{n}, \gamma_{n}^{T} \varphi_{n}(t)\right) z_{n}-\widehat{W}_{n}^{T} S_{n}\left(E_{n}, \widehat{\gamma}_{n}^{T} \varphi_{n}(t)\right) z_{n}+\varepsilon_{n} z_{n}-\widehat{\theta}_{n} \frac{z_{n}^{3} v_{n}^{2}}{\sqrt{z_{n}^{4} v_{n}^{2}+\mu^{2}}}\right. \\
& \left.-\frac{z_{n} \widehat{N}_{n}^{2}}{\sqrt{z_{n}^{2} \widehat{N}_{n}^{2}+\mu^{2}}}+d_{n}(t)\right]-\frac{1}{\eta_{n}} \widetilde{\theta}_{n} \dot{\hat{\theta}}_{n}-\widetilde{W}_{n}^{T} \Gamma_{w_{n}}^{-1} \dot{\hat{W}}_{n}-\operatorname{tr}\left\{\widetilde{\gamma}_{n}^{T} \Gamma_{\gamma_{n}}^{-1} \dot{\hat{\gamma}}_{n}\right\}-\frac{1}{\beta_{n}} \widetilde{N}_{n} \dot{\hat{\hat{N}}}_{n}+\mu+z_{n} L_{\mathrm{st}}\left(\varrho_{n}\right) \bar{\alpha}_{n}-z_{n} \bar{\alpha}_{n} .
\end{aligned}
$$

According to Lemma 2, we have

$$
\begin{aligned}
\dot{V}_{n} \leq & \dot{V}_{n-1}+z_{n}\left[\left(-k_{n}+\frac{1}{2}\right) z_{n}+\widetilde{W}_{n}^{T}\left(\widehat{S}_{n}-\widehat{S}_{n}^{\prime} \widehat{\hat{\gamma}}_{n}^{T} \varphi_{n}(t)\right) z_{n}+\widehat{W}_{n}^{T} \widehat{S}_{n}^{\prime} \tilde{\gamma}_{n}^{T} \varphi_{n}(t) z_{n}+b_{n} z_{n}+\varepsilon_{n} z_{n}\right. \\
& \left.\quad-\widehat{\theta}_{n} \frac{z_{n}^{3} v_{n}^{2}}{\sqrt{z_{n}^{4} v_{n}^{2}+\mu^{2}}}-\frac{z_{n} \widehat{N}_{n}^{2}}{\sqrt{z_{n}^{2} \widehat{N}_{n}^{2}+\mu^{2}}}+d_{n}(t)\right]-\frac{1}{\eta_{n}} \widetilde{\theta}_{n} \dot{\hat{\theta}}_{n}-\widetilde{W}_{n}^{T} \Gamma_{w_{n}}^{-1} \dot{\hat{W}}_{n}-\operatorname{tr}\left\{\widetilde{\gamma}_{n}^{T} \Gamma_{\gamma_{n}}^{-1} \dot{\hat{\gamma}}_{n}\right\}-\frac{1}{\beta_{n}} \widetilde{N}_{n} \dot{\hat{N}}_{n}+\mu+z_{n} L_{\mathrm{st}}\left(\Theta_{n}\right) \bar{u}-z_{n} \bar{u}
\end{aligned}
$$

By using Lemmas 2 and 3, we can get

$$
\begin{aligned}
\left(b_{n}+\varepsilon_{n}\right) z_{n}^{2} \leq & {\left[\left\|\gamma_{n}\right\|_{F}\left\|\varphi_{n}(t) \widehat{W}_{n}^{T} \widehat{S}_{n}^{\prime}\right\|_{F}+\left\|W_{n}\right\|\left\|\varphi_{n}(t) \widehat{\gamma}_{n}^{T} \widehat{S}_{n}^{\prime}\right\|\right.} \\
& \left.+\left|W_{n}\right|_{1}+\left|\varepsilon_{n}\right|\right] z_{n}^{2} \\
\leq & \theta_{n}\left|z_{n}^{2} v_{n}\right| \\
\leq & \theta_{n} \frac{z_{n}^{4} v_{n}^{2}}{\sqrt{z_{n}^{4} v_{n}^{2}+\mu^{2}}}+\theta_{n} \mu,
\end{aligned}
$$

$$
\begin{aligned}
z_{n} d_{n}(t) & \leq\left|z_{n} \| d_{n}(t)\right| \\
& \leq\left|z_{n}\right| N_{n}=\left|z_{n}\right| \widehat{N}_{n}+\left|z_{n}\right| \widetilde{N}_{n} \\
& \leq \frac{z_{n}^{2} \widehat{N}_{n}^{2}}{\sqrt{z_{n}^{2} \widehat{N}_{n}^{2}+\mu^{2}}}+\mu+\left|z_{n}\right| \widetilde{N}_{n},
\end{aligned}
$$

where

$$
\begin{aligned}
& \theta_{n}=\sqrt{\left\|\gamma_{n}\right\|_{F}^{2}+\left\|W_{n}\right\|^{2}+\left(\left|W_{n}\right|_{1}+\left|\varepsilon_{n}\right|\right)^{2}}, \\
& v_{n}=\sqrt{\left\|\varphi_{n}(t) \widehat{W}_{n}^{T} \widehat{S}_{n}^{\prime}\right\|_{F}^{2}+\left\|\varphi_{n}(t) \widehat{\gamma}_{n}^{T} \widehat{S}_{n}^{\prime}\right\|^{2}+1} .
\end{aligned}
$$

$$
\begin{aligned}
\dot{V}_{n} \leq & \dot{V}_{n-1}+z_{n}\left[-\left(k_{n}+\frac{1}{2}\right) z_{n}+\widetilde{W}_{n}^{T}\left(\widehat{S}_{n}-\widehat{S}_{n}^{\prime} \widehat{\gamma}_{n}^{T} \varphi_{n}(t)\right) z_{n}+\widehat{W}_{n}^{T} \widehat{S}_{n}^{\prime} \widetilde{\gamma}_{n}^{T} \varphi_{n}(t) z_{n}-\widetilde{\theta}_{n} \frac{z_{n}^{3} v_{n}^{2}}{\sqrt{z_{n}^{4} v_{n}^{2}+\mu^{2}}}\right] \\
& +\left(\theta_{n}+2\right) \mu+\left|z_{n}\right| \widetilde{N}_{n}-\frac{1}{\eta_{n}} \tilde{\theta}_{n} \dot{\hat{\theta}}_{n}-\widetilde{W}_{n}^{T} \Gamma_{w_{n}}^{-1} \dot{\hat{W}}_{n}-\operatorname{tr}\left\{\widetilde{\gamma}_{n}^{T} \Gamma_{\gamma_{n}}^{-1} \dot{\hat{\gamma}}_{n}\right\}-\frac{1}{\beta_{n}} \widetilde{N}_{n} \dot{\hat{N}}_{n} \\
& +z_{n} L_{\text {st }}\left(\Phi_{n}\right) \bar{u}-z_{n} \bar{u},
\end{aligned}
$$


where $\widehat{W}_{n}^{T} \widehat{S}_{n}{ }^{\prime} \widetilde{\gamma}_{n}^{T} \varphi_{n}(t)=\operatorname{tr}\left\{\widehat{W}_{n}^{T} \widehat{S}_{n}^{\prime} \widetilde{\gamma}_{n}^{T} \varphi_{n}(t)\right\}$. Then, we can get the following inequality:

$$
\begin{aligned}
\dot{V}_{n} \leq & \dot{V}_{n-1}-\left(k_{n}+\frac{1}{2}\right) z_{n}+\left(\theta_{n}+2\right) \mu+z_{n} L_{\mathrm{st}}\left(\Phi_{n}\right) \bar{u}_{n}-z_{n} \bar{u}+\widetilde{W}_{n}^{T}\left[z_{n}^{2}\left(\widehat{S}_{n}-\widehat{S}_{n}^{\prime} \widehat{\hat{\gamma}}_{n}^{T} \varphi_{n}(t)\right)\right. \\
& \left.-\Gamma_{w_{n}}^{-1} \dot{\hat{W}}_{n}\right]+\tilde{\theta}_{n}\left(\frac{z_{n}^{4} v_{n}^{2}}{\sqrt{z_{n}^{4} v_{n}^{2}+\mu^{2}}}-\frac{1}{\eta_{n}} \dot{\hat{\theta}}_{n}\right)+\widetilde{N}_{n}\left(\left|z_{n}\right|-\frac{1}{\beta_{n}} \dot{\hat{N}}_{n}\right)+\operatorname{tr}\left\{\widetilde{\gamma}_{n}^{T}\left(\widehat{W}_{n}^{T} \widehat{S}_{n}^{\prime} \varphi_{n}(t) z_{n}^{2}-\Gamma_{\gamma_{n}}^{-1} \dot{\hat{\gamma}}_{n}\right)\right\} .
\end{aligned}
$$

Based on (71), the adaptive laws are constructed as follows:

$$
\left\{\begin{array}{l}
\dot{\hat{\theta}}_{n}=\eta_{n} \frac{z_{n}^{4} v_{n}^{2}}{\sqrt{z_{n}^{4} v_{n}^{2}+\mu^{2}}}-\rho_{n} \widehat{\theta}_{n}, \\
\dot{\hat{W}}_{n}=\Gamma_{W_{n}}\left[z_{n}^{2}\left(\widehat{S}_{n}-\widehat{S}_{n}^{\prime} \widehat{\gamma}_{n}^{T} \varphi_{n}(t)\right)-\sigma_{n} \widehat{W}_{n}\right] \\
\dot{\hat{\gamma}}_{n}=\Gamma_{\gamma_{n}}\left[z_{n}^{2} \widehat{W}_{n}^{T} \widehat{S}_{n}^{\prime} \varphi_{n}(t)-\kappa_{n} \widehat{\gamma}_{n}\right] \\
\dot{\hat{\hat{N}}}_{n}=\beta_{n}\left|z_{n}\right|-o_{n} \widehat{N}_{n}, \\
\dot{\Phi}_{n}=-D_{n} z_{n} \bar{\alpha}_{n},
\end{array}\right.
$$

where $\rho_{n}, \sigma_{n}, \kappa_{n}, o_{n}$, and $D_{n}$ are positive design parameters. Substituting (72) into (71), the following inequality can be obtained:

$$
\begin{aligned}
\dot{V}_{n} \leq & \dot{V}_{n-1}-\left(k_{n}+\frac{1}{2}\right) z_{n}^{2}+\left(\theta_{n}+2\right) \mu+\frac{\rho_{n}}{\eta_{n}} \widehat{\theta}_{n} \widehat{\theta}_{n}+\sigma_{n} \widetilde{W}_{n}^{T} \widehat{W}_{n}+\kappa_{n} \operatorname{tr}\left\{\widetilde{\gamma}_{n}^{T} \widehat{\gamma}_{n}\right\} \\
& +\frac{o_{n}}{\beta_{n}} \widetilde{N}_{n} \widehat{N}_{n}+\frac{1}{D_{n}} \dot{\oplus}_{n}(t)-\frac{1}{D_{n}} L_{\mathrm{st}}\left(\Phi_{n}(t)\right) \dot{\oplus}_{n}(t) .
\end{aligned}
$$

Using Young's inequality, the following inequalities hold:

$$
\left\{\begin{array}{l}
\frac{\rho_{n}}{\eta_{n}} \widetilde{\theta}_{n} \widehat{\theta}_{n} \leq-\frac{\rho_{n}}{2 \eta_{n}} \widetilde{\theta}_{n}^{2}+\frac{\rho_{n}}{2 \eta_{n}} \theta_{n}^{2}, \\
\sigma_{n} \widetilde{W}_{n}^{T} \widehat{W}_{n} \leq-\frac{\sigma_{n}}{2}\left\|\widetilde{W}_{n}\right\|^{2}+\frac{\sigma_{n}}{2}\left\|W_{n}\right\|^{2}, \\
\kappa_{n} \operatorname{tr}\left\{\tilde{\gamma}_{n}^{T} \widehat{\gamma}_{n}\right\} \leq-\frac{\kappa_{n}}{2}\left\|\widetilde{\gamma}_{n}\right\|_{F}^{2}+\frac{\kappa_{n}}{2}\left\|\gamma_{n}\right\|_{F}^{2}, \\
\frac{o_{n}}{\beta_{n}} \widetilde{N}_{n} \widehat{N}_{n} \leq-\frac{o_{n}}{2 \beta_{n}} \widetilde{N}_{n}^{2}+\frac{o_{n}}{2 \beta_{n}} N_{n}^{2} .
\end{array}\right.
$$

$$
\begin{aligned}
\dot{V}_{n} \leq & -\sum_{j=1}^{n} k_{j} z_{j}^{2}-\sum_{j=1}^{n} \frac{\rho_{j}}{2 \eta_{j}} \widetilde{\theta}_{j}^{2}-\sum_{j=1}^{n} \frac{\sigma_{j}}{2}\left\|\widetilde{W}_{j}\right\|^{2}-\sum_{j=1}^{n} \frac{\kappa_{j}}{2}\left\|\widetilde{\gamma}_{j}\right\|_{F}^{2}-\sum_{j=1}^{n} \frac{o_{j}}{2 \beta_{j}} \widetilde{N}_{j}^{2} \\
& +A_{n}+\sum_{j=1}^{n} \frac{1}{D_{j}} \dot{\boldsymbol{\omega}}_{j}(t)-\sum_{j=1}^{n} \frac{1}{D_{j}} L_{\mathrm{st}}\left(\Phi_{j}(t)\right) \dot{\boldsymbol{\omega}}_{j}(t),
\end{aligned}
$$


where

$$
\begin{aligned}
A_{n}= & \sum_{j=1}^{n}\left(\theta_{j}+2\right) \mu+\sum_{j=1}^{n} \frac{\rho_{j}}{2 \eta_{j}} \theta_{j}^{2}+\sum_{j=1}^{n} \frac{\sigma_{j}}{2}\left\|W_{j}\right\|^{2}+\sum_{j=1}^{n} \frac{\kappa_{j}}{2}\left\|\gamma_{j}\right\|_{F}^{2} \\
& +\sum_{j=1}^{n} \frac{o_{j}}{2 \beta_{j}} N_{j}^{2} .
\end{aligned}
$$

For boundary layer error $e_{i}=s_{i}-\alpha_{i}, i=1, \ldots, n-1$, using the method of differentiation, we have

$$
\begin{gathered}
\dot{e}_{i}=-e_{i}-\frac{\widehat{M}_{i}^{2} e_{i}}{\sqrt{\widehat{M}_{i}^{2} e_{i}+\mu^{2}}}+G_{i}\left(z_{i}, \ldots, z_{i+1}, e_{1}, \ldots, e_{i}, \widehat{\theta}_{1}, \ldots, \widehat{\theta}_{i+1},\right. \\
\left.\widehat{W}_{1}, \ldots, \widehat{W}_{i+1}, \widehat{\gamma}_{1}, \ldots, \widehat{\gamma}_{i+1}, \widehat{M}_{1}, \ldots, \widehat{M}_{i}, y_{r}, \dot{y}_{r}, \ddot{y}_{r}\right),
\end{gathered}
$$

$G_{1}(\cdot)$ and $G_{i}(\cdot)$ are continuous functions and defined as

$$
\begin{aligned}
& G_{1}(\cdot)=-\alpha_{1} \\
& =-\frac{\partial \alpha_{1}}{\partial x_{1}} \dot{x}_{1}-\frac{\partial \alpha_{1}}{\partial \widehat{\hat{\theta}}_{1}} \dot{\hat{\theta}}_{1}-\frac{\partial \alpha_{1}}{\partial \widehat{W}_{1}} \dot{\hat{W}}_{1}-\frac{\partial \alpha_{1}}{\partial \widehat{\gamma}_{1}} \dot{\hat{\gamma}}_{1}-\frac{\partial \alpha_{1}}{\partial y_{d}} \dot{y}_{d}-\frac{\partial \alpha_{1}}{\partial \hat{y}_{d}} \ddot{y}_{d}, \\
& G_{i}(\cdot)=-\alpha_{i} \\
& =-\sum_{j=1}^{i} \frac{\partial \alpha_{i}}{\partial x_{j}} \dot{x}_{j}-\frac{\partial \alpha_{i}}{\partial \widehat{\hat{\theta}}_{i}}-\frac{\partial \alpha_{i}}{\partial e_{i-1}} \dot{e}_{i-1}-\frac{\partial \alpha_{i}}{\partial \widehat{M}_{i-1}} \dot{\hat{M}}_{i-1}-\frac{\partial \alpha_{i}}{\partial \widehat{W}_{i}} \dot{\hat{W}}_{i}-\frac{\partial \alpha_{i}}{\partial \widehat{\hat{\gamma}}_{i}} \dot{\hat{\gamma}}_{i} \\
& -\frac{\partial \alpha_{i}}{\partial y_{r}} \dot{y}_{r}-\frac{\partial \alpha_{i}}{\partial \hat{y}_{r}} \ddot{y}_{r}, \quad i=2, \ldots, n-1
\end{aligned}
$$

The compact sets $\Omega_{1}$ and $\Omega_{2}$ are defined as follows:

$$
\begin{aligned}
& \Omega_{1}=\left\{\left[y_{d}, \dot{y}_{d}, \ddot{y}_{d}\right]^{T}: y_{d}^{2}+\dot{y}_{d}^{2}+\ddot{y}_{d}^{2} \leq G_{0}\right\}, \\
& \Omega_{2}=\{V(t) \leq c\},
\end{aligned}
$$

where $G_{0}$ is a positive constant. It is noting that the set $\Omega_{1} \times \Omega_{2}$ is compact in $R^{4 n+1}$. There is an unknown positive number $M_{i}$ on $\Omega_{1} \times \Omega_{2}$, such that $\left|G_{i}\right| \leq M_{i}$. Therefore, according to (71) and (72), we use its estimated value $\widehat{M}_{i}, i=1, \ldots, n-1$.

The Lyapunov function $V$ is constructed as follows:

$$
V=V_{n}+\sum_{i=1}^{n-1} \frac{1}{2} e_{i}^{2}+\sum_{i=1}^{n-1} \frac{1}{2 \xi_{i}} \tilde{M}_{i}^{2}
$$

where $\xi_{i}$ is the design parameter. Then, the derivative of $V$ is

$$
\begin{aligned}
\dot{V} & =\dot{V}_{n}+\sum_{i=1}^{n-1} e_{i} \dot{e}_{i}-\sum_{i=1}^{n-1} \frac{1}{\xi_{i}} \widetilde{M}_{i} \dot{\bar{M}}_{i} \\
& \leq \dot{V}_{n}-\sum_{i=1}^{n-1} e_{i}^{2}-\sum_{i=1}^{n-1} \frac{\widehat{M}_{i}^{2} e_{i}^{2}}{\sqrt{\hat{M}_{i}^{2} e_{i}^{2}+\mu^{2}}}+\sum_{i=1}^{n-1} M_{i}\left|e_{i}\right|-\sum_{i=1}^{n-1} \frac{1}{\xi_{i}} \widetilde{M}_{i} \dot{\hat{M}}_{i}
\end{aligned}
$$

According to Lemma 3, we have

$$
\begin{aligned}
M_{i}\left|e_{i}\right| & =\widehat{M}_{i}\left|e_{i}\right|+\tilde{M}_{i}\left|e_{i}\right| \\
& \leq \frac{\widehat{M}_{i}^{2} e_{i}^{2}}{\sqrt{\widehat{M}_{i}^{2} e_{i}^{2}+\mu^{2}}}+\mu+\widetilde{M}_{i}\left|e_{i}\right| .
\end{aligned}
$$

Substituting (82) into (81), the following inequality holds:

$\dot{V} \leq \dot{V}_{n}-\sum_{i=1}^{n-1} e_{i}^{2}+(n-1) \mu-\sum_{i=1}^{n-1} \frac{1}{\xi_{i}} \widetilde{M}_{i}\left(\dot{\hat{M}}_{i}-\xi_{i}\left|e_{i}\right|\right)$.

The adaptive law is constructed as follows:

$$
\dot{\hat{M}}_{i}=\xi_{i}\left|e_{i}\right|-\bar{\xi}_{i} \widehat{M}_{i}, \quad i=1, \ldots, n-1,
$$

where $\xi_{i}$ and $\bar{\xi}_{i}$ are the positive parameters. Substituting (84) into (83), the following inequality holds:

$$
\dot{V} \leq \dot{V}_{n}-\sum_{i=1}^{n-1} e_{i}^{2}+\sum_{i=1}^{n-1} \frac{\bar{\xi}_{i}}{\xi_{i}} \widetilde{M}_{i} \widehat{M}_{i}+(n-1) \mu .
$$

Using Young's inequality, the following inequality holds:

$$
\widetilde{M}_{n} \widehat{M}_{n} \leq-\frac{1}{2} \widetilde{M}_{n}^{2}+\frac{1}{2} M_{n}^{2}, \quad i=1, \ldots, n-1 .
$$

Substituting (86) into (85) produces 


$$
\begin{aligned}
& \dot{V} \leq-\left(\sum_{j=1}^{n} k_{j} z_{j}^{2}+\sum_{j=1}^{n} \frac{\rho_{j}}{2 \eta_{j}} \tilde{\theta}_{j}^{2}+\sum_{j=1}^{n} \frac{\sigma_{j}}{2}\left\|\widetilde{W}_{j}\right\|^{2}+\sum_{j=1}^{n} \frac{\kappa_{j}}{2}\left\|\widetilde{\gamma}_{j}\right\|_{F}^{2}+\sum_{j=1}^{n} \frac{o_{j}}{2 \beta_{j}} \widetilde{N}_{j}^{2}+\sum_{j=1}^{n-1} \frac{\bar{\xi}_{j}}{2 \xi_{j}} \tilde{M}_{n}^{2}\right) \\
& +\sum_{j=1}^{n-1} \frac{\bar{\xi}_{j}}{2 \xi_{j}} M_{n}^{2}+(n-1) \mu+A_{n}+\sum_{j=1}^{n} \frac{1}{D_{j}} \dot{\Phi}_{j}(t)-\sum_{j=1}^{n} \frac{1}{D_{j}} L_{s t}\left(\bigotimes_{j}(t)\right) \dot{\Phi}_{j}(t) \\
& \leq-\varepsilon V+\sum_{j=1}^{n} \frac{1}{D_{j}} \dot{\boldsymbol{\omega}}_{j}(t)-\sum_{j=1}^{n} \frac{1}{D_{j}} L_{\mathrm{st}}\left(\varpi_{j}(t)\right) \dot{\boldsymbol{\omega}}_{j}(t)+\chi,
\end{aligned}
$$

where

$$
\begin{aligned}
& \chi=A_{n}+\sum_{j=1}^{n-1} \frac{\bar{\xi}_{j}}{2 \xi_{j}} M_{n}^{2}+(n-1) \mu, \\
& \varepsilon=\min \left\{2 k_{j}, \rho_{j}, \sigma_{j}, \kappa_{j}, o_{j}, \bar{\xi}_{j}\right\} .
\end{aligned}
$$

By integrating (87), we can get the following inequality:

$$
\begin{aligned}
V(t) \leq & -e^{-\varepsilon t} \int_{t_{0}}^{t} \sum_{j=1}^{n} D_{j} L_{\mathrm{st}}\left(\Phi_{j}(\iota)\right) \dot{\boldsymbol{\omega}}_{j}(\iota) e^{\varepsilon \iota} \mathrm{d} \iota \\
& -e^{-\varepsilon t} \int_{t_{0}}^{t} \sum_{j=1}^{n} \frac{1}{D_{j}} \dot{\boldsymbol{\omega}}_{j}(\iota) e^{\varepsilon \iota} \mathrm{d} \iota+\frac{\chi}{\varepsilon}
\end{aligned}
$$

where $(\chi / \varepsilon)$ is a bounded variable and $V(0) e^{-\varepsilon t}$ is bounded. Then, applying Lemma 4 to (89), we can know that all signals in the closed-loop system are bounded. Then, based on (63), we can get $|u(t-\tau(t))-u| \leq \sigma_{\Delta}$, where $\sigma_{\Delta}$ is a positive constant. Let Lyapunov function

$$
V(\lambda)=\frac{1}{2} \sum_{j=1}^{n} \lambda_{j}^{2}
$$

According to (18), the derivative of $V(\lambda)$ can be obtained as follows:

$$
\begin{aligned}
\dot{V}(\lambda) & \leq \sum_{j=2}^{n} \lambda_{j}\left(\lambda_{j+1}-p_{j} \lambda_{j}\right)+\lambda_{n}\left(-p_{n} \lambda_{n}+u(t-\tau(t))-u(t)\right) \\
& \leq \sum_{j=2}^{n} \bar{p}_{j} \lambda_{j}^{2}+\frac{\sigma_{\Delta}}{2} \\
& \leq \sum_{j=2}^{n} \bar{p}_{j} \lambda_{j}^{2}+\sigma_{\lambda}
\end{aligned}
$$

from Young's inequality $\lambda_{j} \lambda_{j+1} \leq(1 / 2) \lambda_{j}^{2}+(1 / 2) \lambda_{j+1}^{2}$, $\lambda_{n} \sigma_{\Delta} \leq(1 / 2) \lambda_{n}^{2}+(1 / 2) \sigma_{\Delta}^{2}$. It can be concluded from (91) that $\bar{p}_{2}=p_{2}-(1 / 2), \bar{p}_{j}=p_{j}-1, j=2, \ldots, n, \bar{p}_{n}=p_{n}-1, \sigma_{\lambda}=$ $\left(\sigma_{\Delta}^{2} / 2\right)$.

Therefore, it can be shown that $\lambda_{i}$ is bounded by (91). From Lemma 5 and (51), $\widehat{W}_{i}^{T}$ is nonnegative. Therefore, we can get the following result:

$$
\begin{aligned}
\dot{\oplus}_{i}(t) & =D_{i}\left(k_{i}+\frac{3}{2}\right) z_{i}^{2}+D_{i} \widehat{W}_{i}^{T} S_{i}\left(E_{i}, \widehat{\gamma}_{i}^{T} \varphi_{i}(t)\right) z_{i}^{2}+\widehat{\theta}_{i} \frac{D_{i} z_{i}^{4} v_{i}^{2}}{\sqrt{z_{i}^{4} v_{i}^{2}+\mu^{2}}}+\frac{D_{i} z_{i}^{2} \widehat{N}_{i}^{2}}{\sqrt{z_{i}^{2} \widehat{N}_{i}^{2}+\mu^{2}}} \\
& \leq D_{i}\left(k_{i}+\frac{3}{2}\right) z_{i}^{2} .
\end{aligned}
$$

On the interval $[0, t]$, the integral of $(92)$ can be obtained as follows:

$$
\int_{0}^{t} D_{i}\left(k_{i}+\frac{3}{2}\right) z_{i}^{2}(\iota) \mathrm{d}(\iota) \leq \oplus_{i}(t)-\varliminf_{i}(0) .
$$

According to Lemma $4, \Phi_{i}(t)$ is bounded, and using Barbalat's Lemma, we can get

$$
\lim _{t \longrightarrow \infty} z_{i}=0, \quad i=1, \ldots, n .
$$

Furthermore, it can be obtained that

$$
\lim _{t \longrightarrow \infty}\left(z_{1}=y-y_{r}\right)=0 .
$$

Thus, the asymptotic tracking of the closed-loop system is achieved.

To show the design idea of the proposed control scheme, the design procedure for the second-order system is presented in Figure 1.

Remark 2. Compared with the control scheme proposed in this paper, similar design works have also been carried out in [39-41]. The main differences can be summarized as follows. Firstly, the uncertain input-delayed systems with periodic 


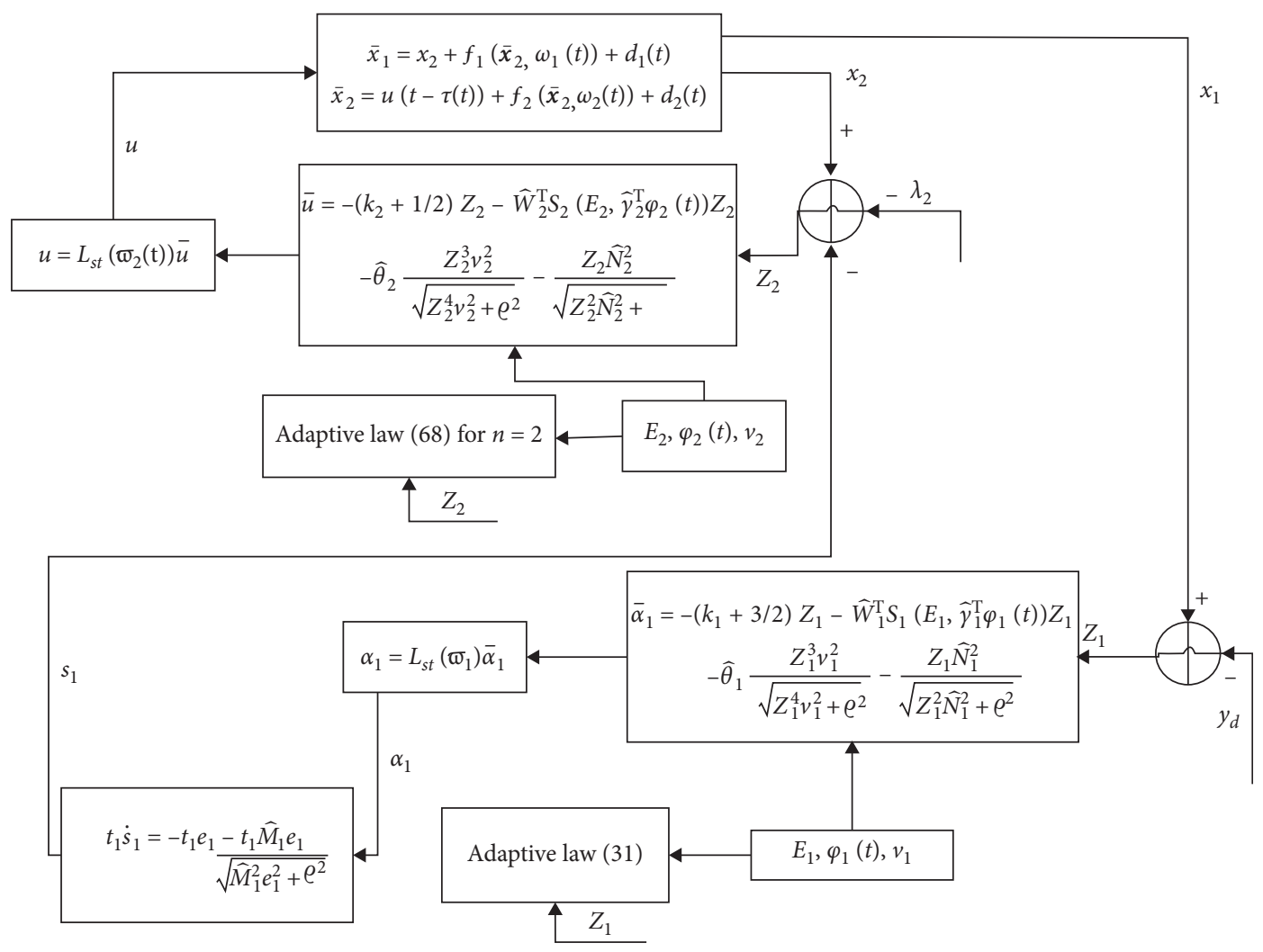

FIGURE 1: Schematic representation of the proposed adaptive neural control scheme.

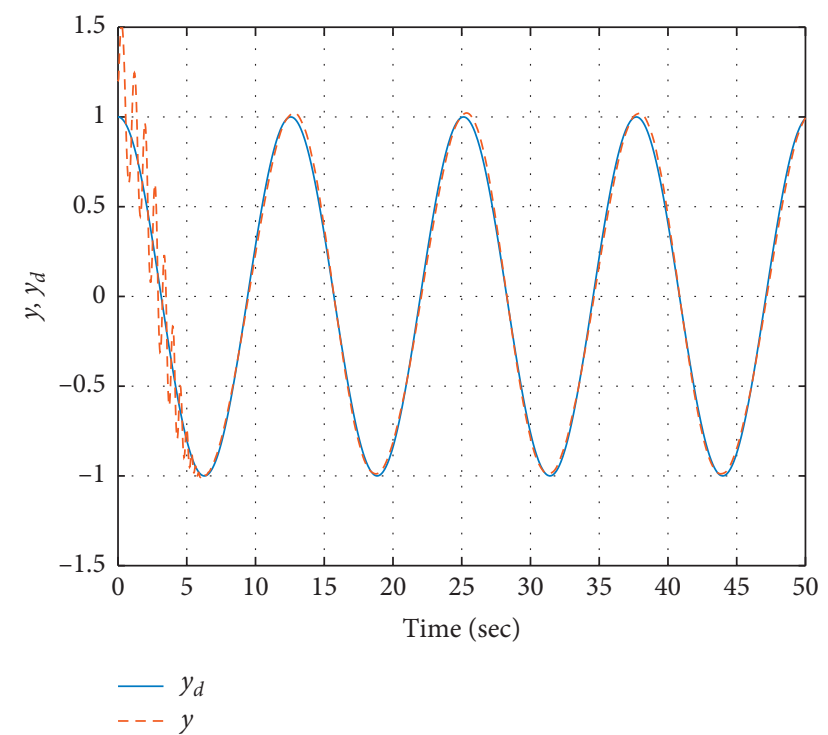

Figure 2: Trajectories of system output signal $y$ and reference signal $y_{d}$.

time-varying disturbances are considered in this paper. Secondly, the problem of adaptive asymptotic tracking control for a class of nonlinear uncertain systems with periodic time-varying disturbances and input delay is solved by developing a new adaptive control scheme based on the FSE-RBFNN approximator. 


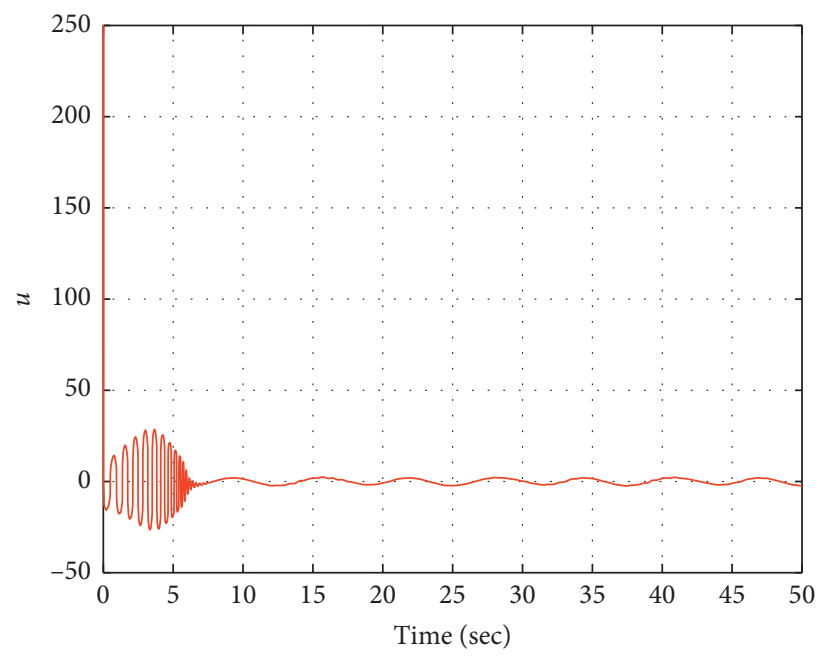

FIgURE 3: Trajectory of control signal $u$.

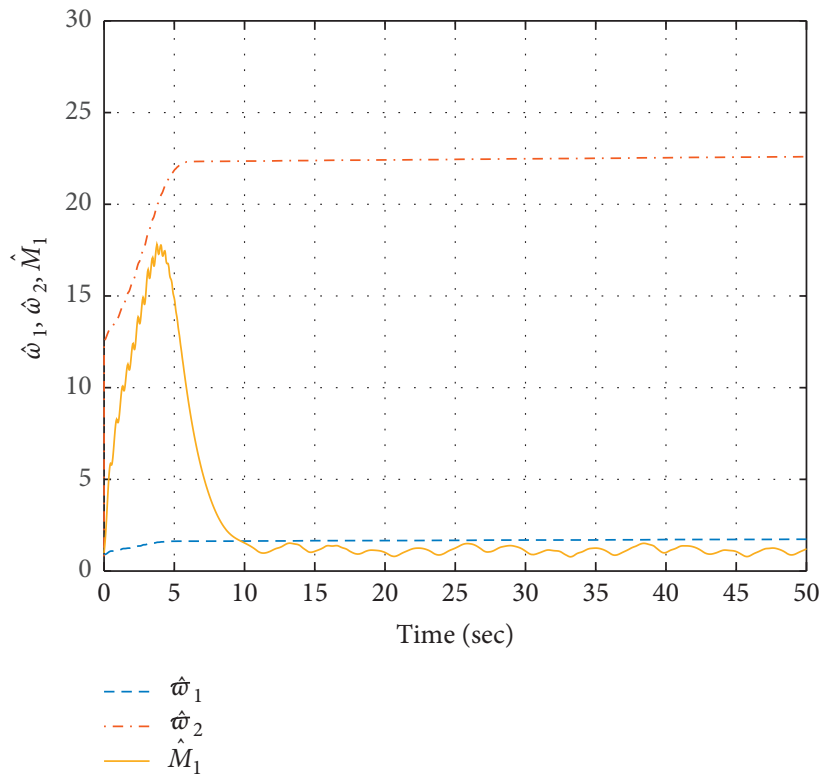

Figure 4: Trajectories of adaptive laws $\widehat{\Phi}_{1}, \widehat{\oplus}_{2}$ and $\widehat{M}_{1}$.

\section{Simulation Example}

In this section, consider the following second-order system:

$$
\left\{\begin{array}{l}
\dot{x}_{1}=x_{2}+\frac{x_{1}^{2} \omega_{1}^{2}+x_{1} \omega_{1}}{x_{1}^{2} \omega_{1}^{2}+10}+\sin (t), \\
\dot{x}_{2}=u(t-\tau(t))+\sin \left(x_{1} x_{2} \omega_{2} e^{-x_{1}^{2} x_{2}^{2} \omega_{2}^{2}}\right)+\cos (t), \\
y=x_{1},
\end{array}\right.
$$

where $\quad \tau=0.04+0.01 \sin (t), \omega_{1}=|\sin (0.5 t)|, \quad$ and $\omega_{2}=|\cos (0.5 t)|$ are unknown time-varying disturbances.
In simulation, the reference signal $y_{d}=\cos (0.5 t)$ and the initial condition of the system is set to be $x_{1}(0)=1.1, x_{2}(0)=0.078, \widehat{\theta}_{1}(0)=0.8, \widehat{\theta}_{2}(0)=0.8 \quad \widehat{M}_{1}(0)$ $=0.99, \Phi_{1}(0)=0.9$, and $\Phi_{2}(0)=0.5$. We choose the numbers of FSE components as $p_{1}=p_{2}=3$. The centres of radial basis functions (RBFs) evenly cover the compact sets $[-4,4] \times[-4,4]$ and $[-2,2] \times[-2,2] \times[-1,1] \times[-1,1]$, and the width of RBFs is 0.9 and the number of nodes of the neural network is 225 . The design parameters are specified as $k_{1}=14.8, k_{2}=39, \eta_{1}=\eta_{2}=5, \rho_{1}=\rho_{2}=5, \sigma_{1}=\sigma_{2}=5, \kappa_{1}=$ $5, \kappa_{2}=10, \beta_{1}=5, \beta_{2}=2, o_{1}=1, o_{2}=2, D_{1}=0.1, D_{2}=7, \mu$ $=5$ and $\xi_{1}=3, \bar{\xi}_{i}=0.6$. The adaptive gains are chosen as $\Gamma_{W_{1}}=\Gamma_{W_{1}}=10 I, \Gamma_{\gamma_{1}}=\Gamma_{\gamma_{1}}=10 I, \widehat{N}_{1}=1$, and $\widehat{N}_{2}=0.1$. The simulation time is 50 seconds, and the CPU time for the proposed algorithm is about 2 minutes for system (96). The simulation results are shown in Figures 2-8. Figure 2 shows 


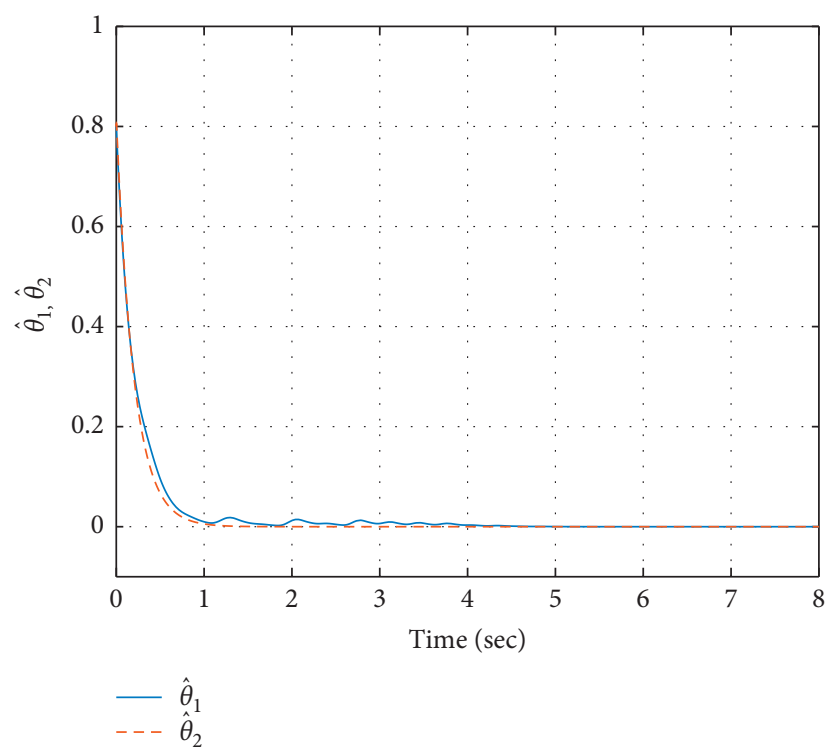

Figure 5: Trajectories of adaptive laws $\widehat{\theta}_{1}$ and $\widehat{\theta}_{2}$.

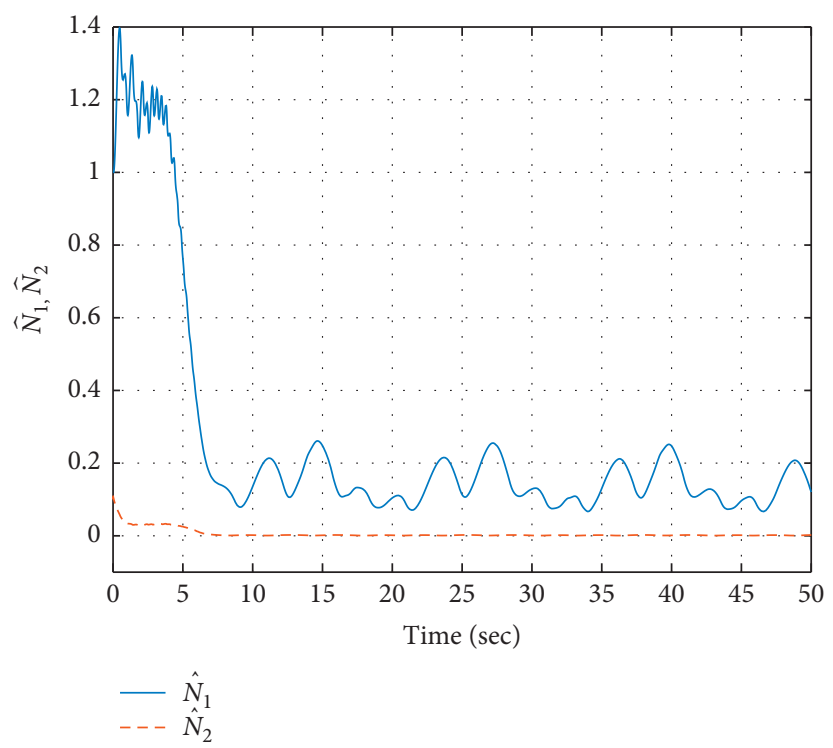

Figure 6: Trajectories of adaptive laws $\widehat{N}_{1}$ and $\widehat{N}_{2}$.

that the system output can asymptotically track the given reference signal, and the boundedness of the closed-loop signals can be seen from Figures 3-8.
Remark 3. To show the superior performance of our control scheme, for system (96), the simulation results by using the method proposed in [32] have been shown in Figures 9 and 


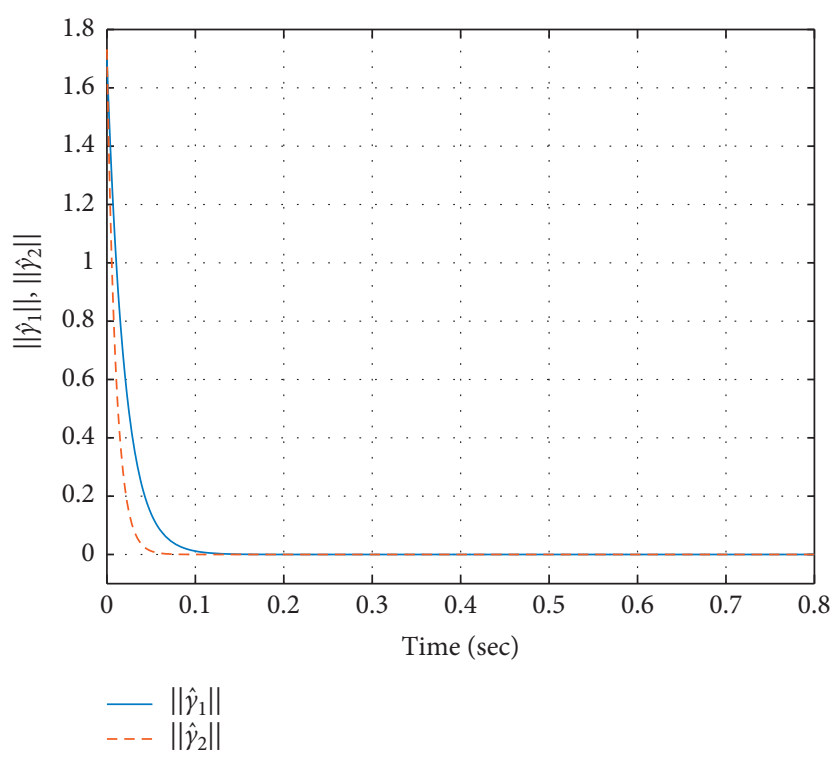

Figure 7: Trajectories of adaptive laws $\left\|\widehat{\gamma}_{1}\right\|$ and $\left\|\widehat{\gamma}_{2}\right\|$.

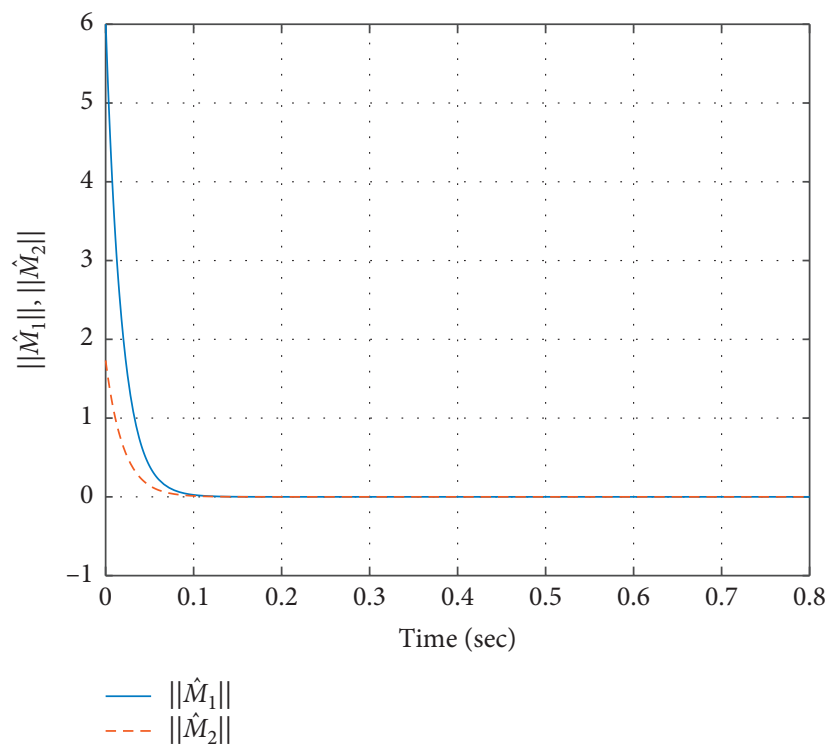

FIgURE 8: Trajectories of adaptive laws $\left\|\widehat{W}_{1}\right\|$ and $\left\|\widehat{W}_{2}\right\|$. 


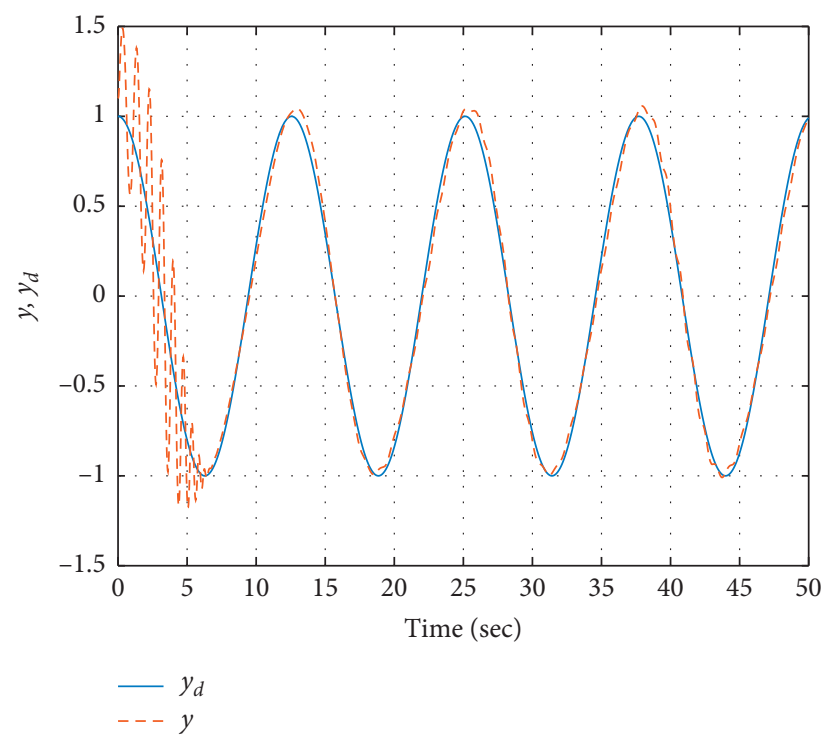

FIgURE 9: Trajectories of system output signal $y$ and reference signal $y_{d}$ using method [32].

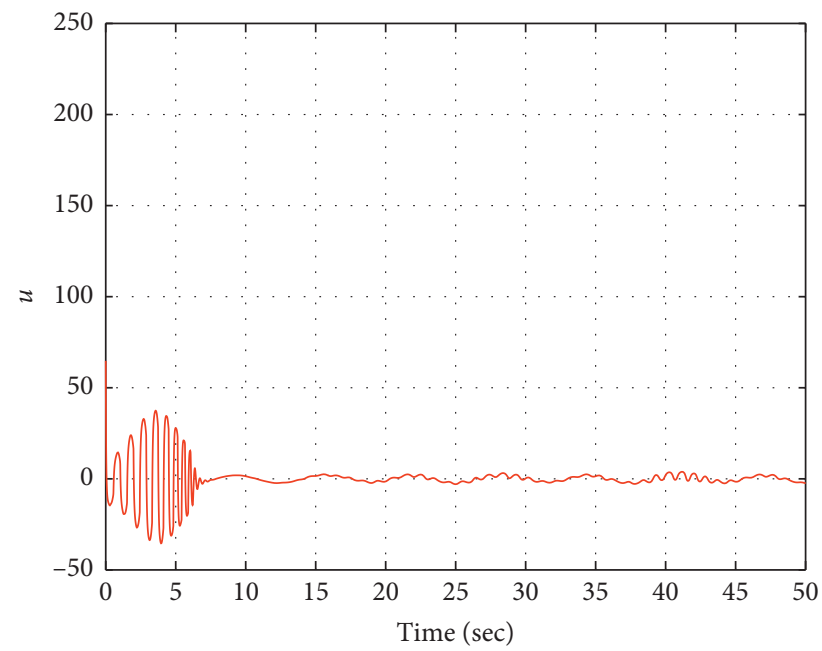

Figure 10: Trajectory of control signal $u$ using method [32].

10. From Figures 2 and 9, it can be seen that the control scheme presented in this paper has a better control performance.

\section{Conclusions}

In this paper, the adaptive asymptotic tracking control for a class of periodic time-varying disturbances uncertain systems with input delay is studied. To solve this problem, a dynamic surface technique based on a hybrid approximator is proposed. It is proved that the tracking error asymptotically converges to zero, and all the closed-loop signals are semiglobally uniformly bounded. Finally, the simulation example validates that the proposed design scheme is feasible. The control scheme proposed in this paper is a theoretical result, and from the simulation example, it can be seen that from the implementation and computational point of view, the feasibility of the proposed approach is guaranteed. However, how to test their technique against a realistic system is a more challenging problem, which is the main disadvantage of the proposed method and will be discussed in further research.

\section{Data Availability}

No data were used to support this study.

\section{Disclosure}

The manuscript has not been published in whole or in part elsewhere. The paper is not currently being considered for publication elsewhere. All authors have been personally and actively involved in substantive work leading to the report and will hold themselves jointly and individually responsible for its content. 


\section{Conflicts of Interest}

The authors declare that they have no conflicts of interest.

\section{Acknowledgments}

This study was funded by the National Natural Science Foundation of China (grant no. 61603003), the Natural Science Foundation of Anhui Province (grant no. 1608085QF131), the Program for Academic Top-Notch Talents of University Disciplines (grant no. gxbjZD21), and the Program for Innovative Research Team in Anqing Normal University.

\section{References}

[1] Y. N. Yang and Y. Yan, "Backstepping sliding mode control for uncertain strict-feedback nonlinear systems using neuralnetwork-based adaptive gain scheduling," Journal of Systems Engineering and Electronics, vol. 29, no. 3, pp. 580-586, 2018.

[2] W. Chen, S. S. Ge, J. Wu, and M. Gong, "Globally stable Adaptive backstepping neural network control for uncertain strict-feedback systems with tracking accuracy known a priori," IEEE Transactions on Neural Networks and Learning Systems, vol. 26, no. 9, pp. 1842-1854, 2015.

[3] L. Liu, Y. J. Liu, S. C. Tong, and C. L. P. Chen, "Integral barrier Lyapunov function based adaptive control for switched nonlinear systems," Science China Information Sciences, vol. 63, no. 3, pp. 132203-1-14, 2020.

[4] J. Wu, B. Su, J. Li, X. Zhang, X. Li, and W. Chen, "Adaptive fuzzy control for full states constrained systems with nonstrict-feedback form and unknown nonlinear dead zone," Information Sciences, vol. 376, pp. 233-247, 2017.

[5] L. Liu, X. Li, Y.-J. Liu, and S. Tong, "Neural network based adaptive event trigger control for a class of electromagnetic suspension systems," Control Engineering Practice, vol. 106, p. $104675,2021$.

[6] H. A. Yousef, M. Hamdy, and K. Nashed, "L1 Adaptive fuzzy controller for a class of nonlinear systems with unknown backlash-like hysteresis," International Journal of Systems Science, vol. 48, no. 12, pp. 2522-2533, 2017.

[7] Y. Yang, J. Tan, and D. Yue, "Prescribed performance tracking control of a class of uncertain pure-feedback nonlinear systems with input saturation," IEEE Transactions on Systems, Man, and Cybernetics: Systems, vol. 50, no. 5, pp. 1733-1745, 2020.

[8] J. Wu, X. Chen, Q. Zhao, J. Li, and Z.-G. Wu, "Adaptive neural dynamic surface control with prespecified tracking accuracy of uncertain stochastic nonstrict-feedback systems," IEEE Transactions on Cybernetics, p. 1, 2020.

[9] M. Chen, S. S. Ge, and B. V. E. How, "Robust adaptive neural network control for a Class of uncertain MIMO nonlinear systems with input nonlinearities," IEEE Transactions on Neural Networks, vol. 21, no. 5, pp. 796-812, 2010.

[10] H. Dong, S. Gao, B. Ning, T. Tang, Y. Li, and K. P. Valavanis, "Error-driven nonlinear feedback design for fuzzy adaptive dynamic surface control of nonlinear systems with prescribed tracking performance," IEEE Transactions on Systems, Man, and Cybernetics: Systems, vol. 50, no. 3, pp. 1013-1023, 2020.

[11] Z. Chen, Z. Li, and C. L. P. Chen, "Adaptive neural control of uncertain MIMO nonlinear systems with state and input constraints," IEEE Transactions on Neural Networks and Learning Systems, vol. 28, no. 6, pp. 1318-1330, 2017.
[12] S. Sun, H. Zhang, Y. Cai, and Y. Mu, "Observer and faulttolerant controller design for discrete-time multiple statedelayed T-S fuzzy systems," IET Control Theory \& Applications, vol. 14, no. 11, pp. 1411-1423, 2020.

[13] X. Xia, G. Kang, T. Zhang, and Y. Fang, “Adaptive quantised control of uncertain non-linear systems with state constraints and time-varying delays," IET Control Theory \& Applications, vol. 14, no. 10, pp. 1308-1320, 2020.

[14] D. Zhai, A. Y. Lu, J. X. Dong, and Q. L. Zhang, "Adaptive fuzzy tracking control for a class of switched uncertain nonlinear systems: an adaptive state-dependent switching law method," IEEE Transactions on Systems, Man, and Cybernetics: Systems, vol. 48, no. 12, pp. 2282-2291, 2017.

[15] L. Long and J. Zhao, "Adaptive output-feedback neural control of switched uncertain nonlinear systems with average dwell time," IEEE Transactions on Neural Networks and Learning Systems, vol. 26, no. 7, pp. 1350-1362, 2015.

[16] L. Lin, Z. Liu, Y. Kao, and R. Xu, "Observer-based adaptive sliding mode control of uncertain switched systems," IET Control Theory \& Applications, vol. 14, no. 3, pp. 519-525, 2020.

[17] X. Zhao, X. Wang, L. Ma, and G. Zong, "Fuzzy approximation based asymptotic tracking control for a class of uncertain switched nonlinear systems," IEEE Transactions on Fuzzy Systems, vol. 28, no. 4, pp. 632-644, 2020.

[18] W. Zou, C. K. Ahn, and Z. Xiang, "Analysis on existence of compact set in neural network control for nonlinear systems," Automatica, vol. 120, p. 109155, 2020.

[19] M. A. Regaieg, M. Kchaou, J. Bosche, A. El-Hajjaji, and M. Chaabane, "Robust dissipative observer-based control design for discrete-time switched systems with time-varying delay," IET Control Theory \& Applications, vol. 13, no. 18, pp. 3026-3039, 2019.

[20] K. Abidi, H. J. Soo, and I. Postlethwaite, "Discrete-time adaptive control of uncertain sampled-data systems with uncertain input delay: a reduction," IET Control Theory \& Applications, vol. 14, no. 13, pp. 1681-1691, 2020.

[21] J. Wu, Z. G. Wu, J. Li, G. Wang, H. Zhao, and W. Chen, "Practical adaptive fuzzy control of nonlinear pure-feedback systems with quantized nonlinearity input," IEEE Transactions on Systems, Man, and Cybernetics: Systems, vol. 49, no. 3, pp. 638-648, 2019.

[22] M. Lv, B. De Schutter, W. Yu, W. Zhang, and S. Baldi, "Nonlinear systems with uncertain periodically disturbed control gain functions: adaptive fuzzy control with invariance properties," IEEE Transactions on Fuzzy Systems, vol. 28, no. 4, pp. 746-757, 2020.

[23] H. Muramatsu and S. Katsura, "An adaptive periodic-disturbance observer for periodic-disturbance suppression," IEEE Transactions on Industrial Informatics, vol. 14, no. 10, pp. 4446-4456, 2018.

[24] W. S. Chen and L. C. Jiao, "Adaptive tracking for periodically time-varying and nonlinearly parameterized systems using multilayer neural networks," IEEE Transactions on Neural Networks, vol. 21, no. 2, pp. 345-351, 2010.

[25] W. Sun, S.-F. Su, J. Xia, and Y. Wu, "Adaptive tracking control of wheeled inverted pendulums with periodic disturbances," IEEE Transactions on Cybernetics, vol. 50, no. 5, pp. 1867$1876,2020$.

[26] S. Li, C. K. Ahn, J. Guo, and Z. Xiang, "Neural-network approximation-based adaptive periodic event-triggered output-feedback control of switched nonlinear systems," IEEE Transactions on Cybernetics, p. 1, 2020. 
[27] W. S. Chen, L. C. Jiao, R. H. Li, and J. Li, "Adaptive backstepping fuzzy control for nonlinearly parameterized systems with periodic disturbances," IEEE Transactions on Fuzzy Systems, vol. 18, no. 4, pp. 674-685, 2010.

[28] S. Li, C. K. Ahn, J. Guo, and Z. Xiang, "Neural network-based sampled-data control for switched uncertain nonlinear systems," IEEE Transactions on Systems, Man, and Cybernetics: Systems, p. 1, 2019.

[29] D. Swaroop, J. K. Hedrick, P. P. Yip, and J. C. Gerdes, "Dynamic surface control for a class of nonlinear systems," IEEE Transactions on Automatic Control, vol. 45, no. 10, pp. 1893-1899, 2000.

[30] H. Liu, Y. Pan, and J. Cao, "Composite learning adaptive dynamic surface control of fractional-order nonlinear systems," IEEE Transactions on Cybernetics, vol. 50, no. 6, pp. 2557-2567, 2020.

[31] M. Chen, G. Tao, and B. Jiang, "Dynamic surface control using neural networks for a class of uncertain nonlinear systems with input saturation," IEEE Transactions on Neural Networks and Learning Systems, vol. 26, no. 9, pp. 2086-2097, 2015.

[32] Z. Ma and H. Ma, "Adaptive fuzzy backstepping dynamic surface control of strict-feedback fractional-order uncertain nonlinear systems," IEEE Transactions on Fuzzy Systems, vol. 28, no. 1, pp. 122-133, 2020.

[33] L. Wang and C. L. P. Chen, "Adaptive fuzzy dynamic surface control of nonlinear constrained systems with unknown virtual control coefficients," IEEE Transactions on Fuzzy Systems, vol. 28, no. 8, pp. 1737-1747, 2020.

[34] F. Shojaei, M. M. Arefi, A. Khayatian, and H. R. Karimi, "Observer-based fuzzy adaptive dynamic surface control of uncertain nonstrict feedback systems with unknown control direction and unknown dead-zone," IEEE Transactions on Systems, Man, and Cybernetics: Systems, vol. 49, no. 11, pp. 2340-2351, 2019.

[35] J. Ma, S. Xu, G. Zhuang, Y. Wei, and Z. Zhang, "Adaptive neural network tracking control for uncertain nonlinear systems with input delay and saturation," International Journal of Robust and Nonlinear Control, vol. 30, no. 7, pp. 2593-2610, 2020.

[36] W. S. Chen, "Adaptive backstepping dynamic surface control for systems with periodic disturbances using neural networks," IET Control Theory \& Applications, vol. 10, no. 3, pp. 1383-1394, 2009.

[37] Z. Zuo and C. Wang, "Adaptive trajectory tracking control of output constrained multi-rotors systems," IET Control Theory \& Applications, vol. 8, no. 13, pp. 1163-1174, 2014.

[38] K. Xie, C. Chen, F. L. Lewis, and S. Xie, "Adaptive asymptotic neural network control of nonlinear systems with unknown actuator quantization," IEEE Transactions on Neural Networks and Learning Systems, vol. 29, no. 12, pp. 6303-6312, 2018.

[39] S. Sui, S. Tong, and C. L. P. Chen, "Finite-time filter decentralized control for nonstrict-feedback nonlinear largescale systems," IEEE Transactions on Fuzzy Systems, vol. 26, no. 6, pp. 3289-3300, 2018.

[40] S. Sui, C. L. P. Chen, and S. Tong, "Neural network filtering control design for nontriangular structure switched nonlinear systems in finite time," IEEE Transactions on Neural Networks and Learning Systems, vol. 30, no. 7, pp. 2153-2162, 2019.

[41] S. Sui, C. L. P. Chen, and S. Tong, "Neural-network-based adaptive DSC design for switched fractional-order nonlinear systems," IEEE Transactions on Neural Networks and Learning Systems, p. 1, 2020. 